Article

\title{
Synthesis and Anti-Fungal Activity of Seven Oleanolic Acid Glycosides
}

\section{Hanqing Zhao, Guanghui Zong, Jianjun Zhang *, Daoquan Wang and Xiaomei Liang *}

Key Lab of Pesticide Chemistry and Application Technology, Department of Applied Chemistry, China Agricultural University, Beijing 100193, China

* Authors to whom correspondence should be addressed; E-Mails: zhangjianjun@cau.edu.cn (J.Z.); nmrlab@cau.edu.cn (X.L.); Tel.: +86-10-6273-1115.

Received: 7 December 2010; in revised form: 23 December 2010 / Accepted: 13 January 2011 / Published: 26 January 2011

\begin{abstract}
In order to develop potential anti-fungal agents, seven glycoconjugates composed of $\alpha$-L-rhamnose, 6 -deoxy- $\alpha$-L-talose, $\beta$-D-galactose, $\alpha$-D-mannose, $\beta$-D-xylose$(1 \rightarrow 4)-6$-deoxy- $\alpha$-L-talose, $\beta$-D-galactose- $(1 \rightarrow 4)$ - $\alpha$-L-rhamnose, $\beta$-D-galactose- $(1 \rightarrow 3)-\beta$ D-xylose- $(1 \rightarrow 4)-6$-deoxy- $\alpha$-L-talose as the glycone and oleanolic acid as the aglycone were synthesized in an efficient and practical way using glycosyl trichloroacetimidates as donors. The structures of the new compounds were confirmed by $\mathrm{MS},{ }^{1} \mathrm{H}-\mathrm{NMR}$ and ${ }^{13} \mathrm{C}$ NMR. Preliminary studies based on means of mycelium growth rate, indicated that all the compounds possess certain fungicidal activity against Sclerotinia sclerotiorum (Lib.) de Bary, Rhizoctonia solani Kuhn, Botrytis cinerea Pers and Phytophthora parasitica Dast.
\end{abstract}

Keywords: synthesis; oleanolic acid; glycoconjugate; anti-fungal activity

\section{Introduction}

During the course of growth and development, plants synthesize triterpenoid saponins which act as preformed chemical barriers against fungal attack [1]. Aside from their important role in plant growth, these glycosylated plant secondary metabolites show various kinds of biological activity and have been used widely as anti-inflammatory, anti-tumor, anti-HIV, and antifungal agents [2]. Consequently, triterpenoid saponin structures have become the synthetic targets of many research groups $[3,4]$. One common feature shared by all saponins is the presence of a sugar chain at the C-3 of the aglycone 
moiety $[5,6]$. These chains vary from saponin to saponin but usually consist of glucose, arabinose, glucuronic acid, xylose or rhamnose [7].

Recently Yadava et. al. reported a new triterpenoid saponin isolated from the seeds of $L$. scariola, which had the structure of 3-O-[ $\beta$-D-galactopyranosyl-( $1 \rightarrow 3)-O-\beta$-D-xylopyranosyl- $(1 \rightarrow 4)-O-\alpha-\mathrm{L}-$ rhamnopyranosyl]-oleanolic acid (Figure 1, I) [8]. Interestingly, this triterpenoid saponin exhibited broad spectrum antibacterial and antifungal activities against Staphylococcus aureus, Escherichia coli, Penicillium digitatum and Aspergillus niger [8]. In a project for the discovery of novel environmentally friendly antifungal agents from natural resources, we engaged in the study of the synthesis and anti-fungal activity of glycoconjugate derivatives 1-7. We report herein the preliminary results of the study.

Figure 1. Structure of the triterpenoid saponin (I) and target compounds 1-7.

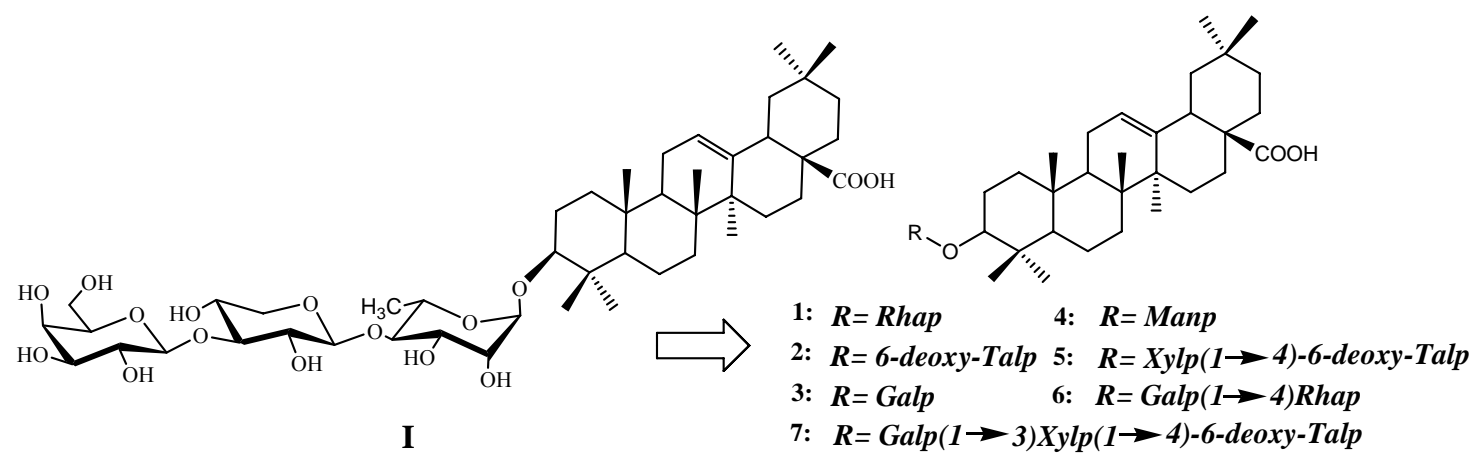

\section{Results and Discussion}

\subsection{Chemistry}

As shown in Figure 2, we envisioned that the target compounds 1-7 could be synthesized using nine suitably protected building blocks 9-16.

Figure 2. The building blocks 9-16 used for the synthesis of target compounds 1-7.

Target compounds 1-7

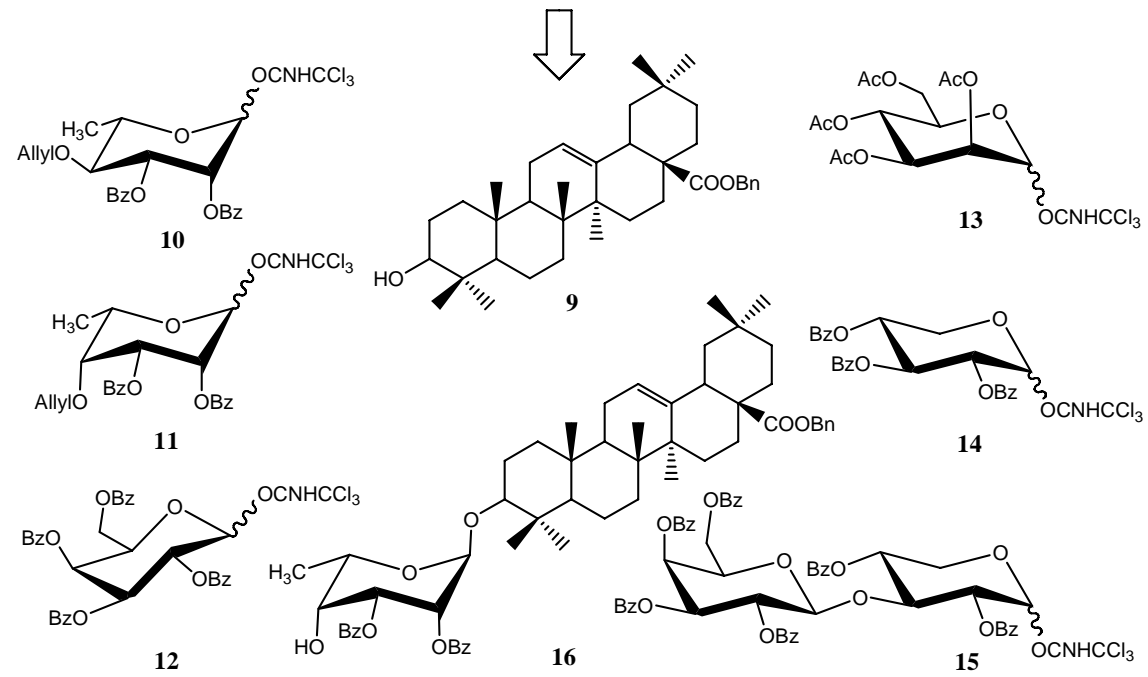


In our work, the Schmidt method [9] was used in the glycosylation, and benzyl was chosen as the protective group for the $\mathrm{COOH}$ group to avoid difficulties in the final deprotection. Since the synthons 9 [10], 12 [11], 13 [12] and 14 [13] were easily prepared according to the reported procedures, our attention was focused on the synthesis of 4-O-allyl-2,3-di- $O$-benzoyl- $\alpha$-L-rhamnopyranosyl trichloroacetimidate (10), 4- $O$-allyl-2,3-di- $O$-benzoyl-6-deoxy- $\alpha$-L-talopyranosyl trichloroacetimidate (11), 2,3,4,6-tetra-O-benzoyl- $\beta$-D-galactopyranose-( $1 \rightarrow 3)-2,4$-di- $O$-benzoyl- $\beta$-D-xylopyranosyl trichloroacetimidate (15) and benzyl oleanolate 3-O-2,3-di-O-benzoyl- $\alpha$-L-talopyranoside (16) (Scheme 1).

Scheme 1. Synthetic routes to the compounds 10, 11, 15 and 16.
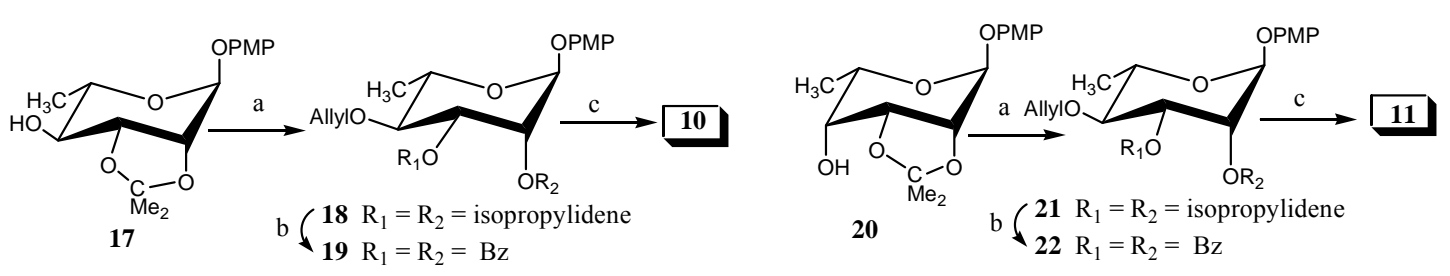

$20 \quad b\left(\begin{array}{ll}21 & R_{1}=R_{2}=\text { isop } \\ 22 & R_{1}=R_{2}=B z\end{array}\right.$
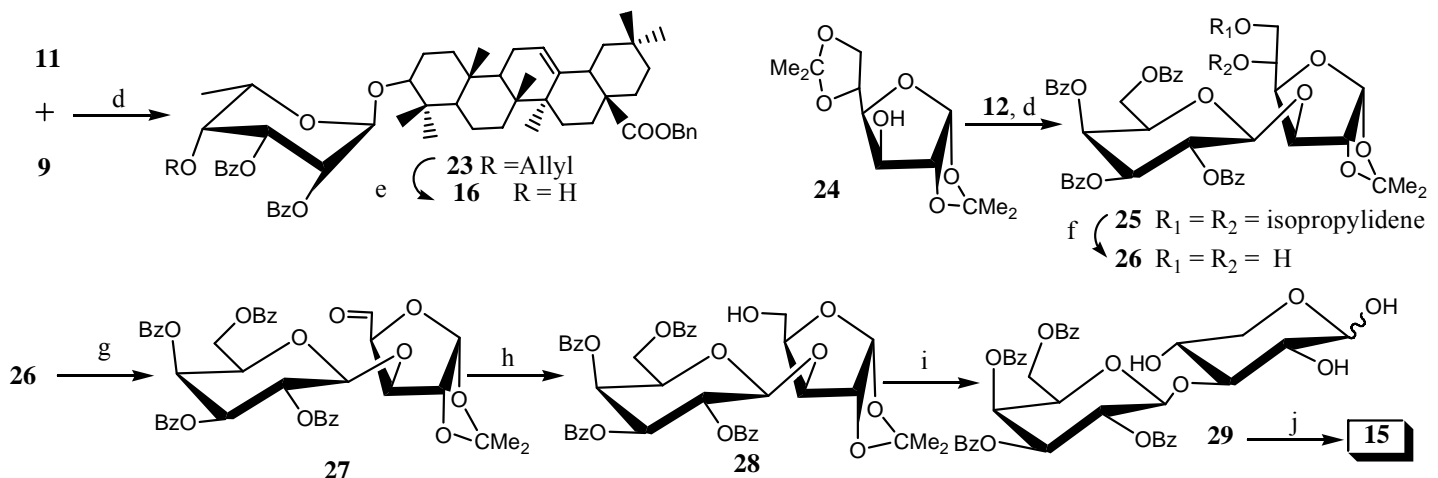

Reagents and conditions: (a) AllBr, NaH, DMF, $0{ }^{\circ} \mathrm{C}, 2 \mathrm{~h}, 95 \%$ for 18, $92 \%$ for 21 ; (b) $70 \% \mathrm{HOAc}$, $70{ }^{\circ} \mathrm{C}, 2 \mathrm{~h}$; then BzCl-Py. $88 \%$ for 19 (2 steps), $80 \%$ for 22 (2 steps); (c) $80 \% \mathrm{MeCN}, \mathrm{CAN}, 35^{\circ} \mathrm{C}$, 20 min; then $\mathrm{CNCCl}_{3}, \mathrm{CH}_{2} \mathrm{Cl}_{2}$, DBU, $4 \mathrm{~h}, 72 \%$ for $\mathbf{1 0}$ (2 steps), 70\% for $\mathbf{1 1}$ (2 steps). (d) TMSOTf, $\mathrm{CH}_{2} \mathrm{Cl}_{2},-10{ }^{\circ} \mathrm{C}$ to r.t., $2 \mathrm{~h}, 85 \%$ for $23,88 \%$ for 25. (e) $\mathrm{MeOH}-\mathrm{CH}_{2} \mathrm{Cl}_{2}=1 / 1, \mathrm{PdCl}_{2}$, r.t. $92 \%$ for 16 . (f) $60 \% \mathrm{HOAc}$, r.t. $83 \%$ for 26. (g) $\mathrm{NaIO}_{4}-\mathrm{SiO}_{2}, \mathrm{CH}_{2} \mathrm{Cl}_{2}, 88 \%$ for 27. (h) $\mathrm{NaBH}_{4}$, EtOAc- $\mathrm{H}_{2} \mathrm{O}=7: 3$, $0{ }^{\circ} \mathrm{C}$ to r.t., $15 \mathrm{~min}, 96 \%$ for 28. (i) $4 \% \mathrm{H}_{2} \mathrm{SO}_{4}$, reflux $3-4 \mathrm{~h}, 85 \%$ for 29. (j) $\mathrm{Py}$, $\mathrm{BzCl}$, then $2 \mathrm{M}$ $\mathrm{MeOH}-\mathrm{NH}_{3}, 2 \mathrm{~h}$, then $\mathrm{CNCCl}_{3}, \mathrm{CH}_{2} \mathrm{Cl}_{2}, \mathrm{DBU}, 4 \mathrm{~h}, 68 \%$ for $\mathbf{1 5}$ (3 steps).

Among these compounds, donor 10 was prepared from the known $p$-methoxyphenyl 2,3-Oisopropylidene- $\alpha$-L-rhamnopyranoside (17) [14]. Allylation of 17 with allyl bromide provided the corresponding $\quad p$-methoxyphenyl 4-O-allyl-2,3- $O$-isopropylidene- $\alpha$-L-rhamnopyranoside 18 quantitatively; then removal of the isopropylidene group with $70 \%$ HOAc followed by benzoylation gave 19 in high yield (88\%); finally, cleavage of the $p$-methoxyphenyl glycoside in 19 with ceric ammonium nitrate (CAN) followed by trichloroacetimidation afforded the corresponding glycosyl donor 10 in 72\% yield. Meanwhile, the donor 11 was prepared in a similar way, i.e., allylation of 20 [15] provided the corresponding $p$-methoxyphenyl 4- $O$-allyl-2,3- $O$-isopropylidene-6-deoxy- $\alpha$-L-talopyranoside 21 quantitatively; and removal of the isopropylidenyl group followed by benzoylation gave 22 in high yield (80\%); finally, cleavage of the $p$-methoxyphenyl glycoside in 22 with ceric ammonium nitrate (CAN) followed by trichloroacetimidation afforded the corresponding glycosyl donor 11 in 70\% yield. Condensation of donor 11 with C-3-OH acceptor 9 [10] in the presence of 
TMSOTf gave the $\alpha$-linked 6-deoxy-taloside 23, whose ${ }^{1} \mathrm{H}$-NMR spectrum showed characteristic signals of a doublet at $\delta 5.42 \mathrm{ppm}\left(J_{1,2}=3.6 \mathrm{~Hz}\right)$ for the $\mathrm{H}-1$ of 6-deoxytalose, a multiplet at $\delta 5.88$ ppm for $\mathrm{CH}_{2}=\mathrm{CH}-\mathrm{CH}_{2} \mathrm{O}$, and seven singlets at $\delta 1.12,1.01,0.92,0.91,0.89,0.83,0.60 \mathrm{ppm}$ for the $\mathrm{CH}_{3}$ groups of oleanolic acid. Deallylation of 23 with $\mathrm{PdCl}_{2}$ gave the desired acceptor 16, and the ${ }^{1} \mathrm{H}-$ NMR showed that the characteristic allyl signals had disappeared.

On the other hand, we have also developed a novel strategy for the synthesis of 2,3,4,6-tetra-Obenzoyl- $\beta$-D-galactopyranose- $(1 \rightarrow 3)-2,4-$ di- $O$-benzoyl- $\beta$-D-xylopyranosyl trichloroacetimidate 15 from 2,3,4,6-tetra- $O$-benzoyl- $\beta$-D-galactopyranosyl trichloroacetimidate $\mathbf{1 2}$ and 1,2:5,6-di- $O$ isopropylidene- $\alpha$-D-glucofuranose 24 [16]. 2,3,4,6-Tetra- $O$-benzoyl- $\beta$-D-galactopyranose-( $1 \rightarrow 3)-1,2-$ $O$-isopropylidene- $\beta$-D-xylose 28 was conveniently prepared from 25 in $70 \%$ overall yield, via selective removal of the 5,6-O-isopropylidene group followed by $\mathrm{NaIO}_{4}$ oxidation and $\mathrm{NaBH}_{4}$ reduction in a similar way as reported in [17]. Subsequently, hydrolysis of 28 was carried out in an aqueous solution of sulfuric acid (4\%) under heating at reflux, and the reaction was accompanied by ring expansion [18] to provide 2,3,4,6-tetra-O-benzoyl- $\beta$-D-galactopyranose- $(1 \rightarrow 3)-\beta$-D-xylose 29 , which was benzoylated with benzoyl chloride in pyridine. Regioselective removal of the 1-O-benzoyl group in 2 $\mathrm{M} \mathrm{MeOH}-\mathrm{NH}_{3}$ followed by trichloroacetimidation with trichloroacetonitrile [9] afforded building block 15 in 68\% yield ( 3 steps). Finally, condensation of the donor 10 with the acceptor $\mathbf{9}$ in the presence of TMSOTf gave benzyl oleanolate 3-O-4-O-allyl-2,3-di- $O$-benzoyl- $\alpha$-L-rhamnopyranoside 30 in $88 \%$ yield (Scheme 2). The structure was confirmed by its ${ }^{1} \mathrm{H}-\mathrm{NMR}$ spectrum, showing characteristic signals at $\delta 4.97 \mathrm{ppm}\left(J_{1,2}=1.7 \mathrm{~Hz}\right)$ for the $\mathrm{H}-1$ of rhamnose, $\delta 5.81 \mathrm{ppm}$ for $\mathrm{CH}_{2}=\mathrm{C} \underline{\mathrm{H}}-$ $\mathrm{CH}_{2} \mathrm{O}$, and $\delta 1.12,1.02,0.92,0.92,0.89,0.88,0.61 \mathrm{ppm}$ for $\mathrm{CH}_{3}$ of oleanolic acid, the ${ }^{13} \mathrm{C}-\mathrm{NMR}$ spectrum showed peaks at $\delta 99.6 \mathrm{ppm}$ for anomeric C-1. Deallylation of $\mathbf{3 0}$ gave the desired acceptor 31 in 94\% yields. The other five oleanolic acid glycosides 34, 36, 38, 40 and 42 were prepared from condensation of the donors and the acceptors 12 and 9,13 and 9, 14 and 16, 12 and 31, 15 and 16 respectively, giving $86 \% \sim 90 \%$ yields.

Scheme 2. Synthesis of the target compounds 1-7.
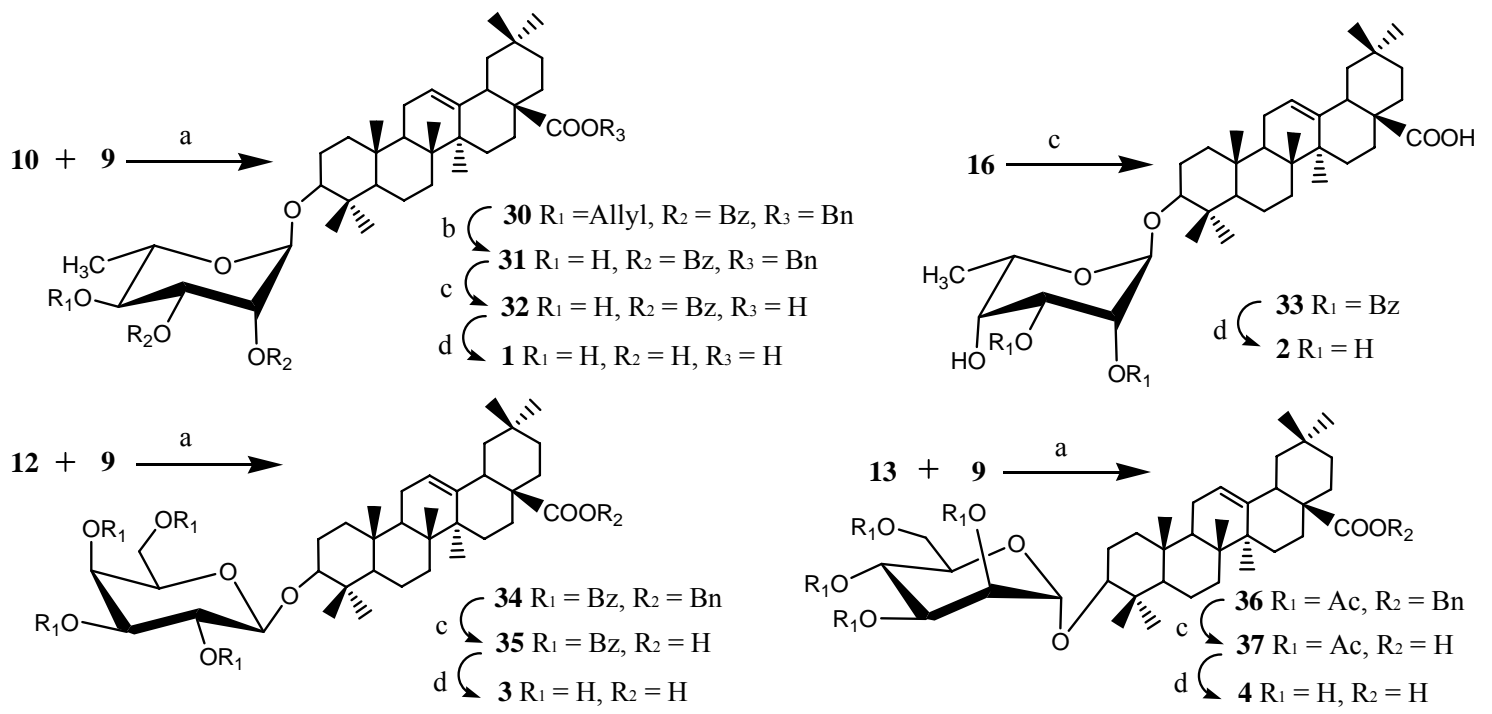
Scheme 2. Cont.

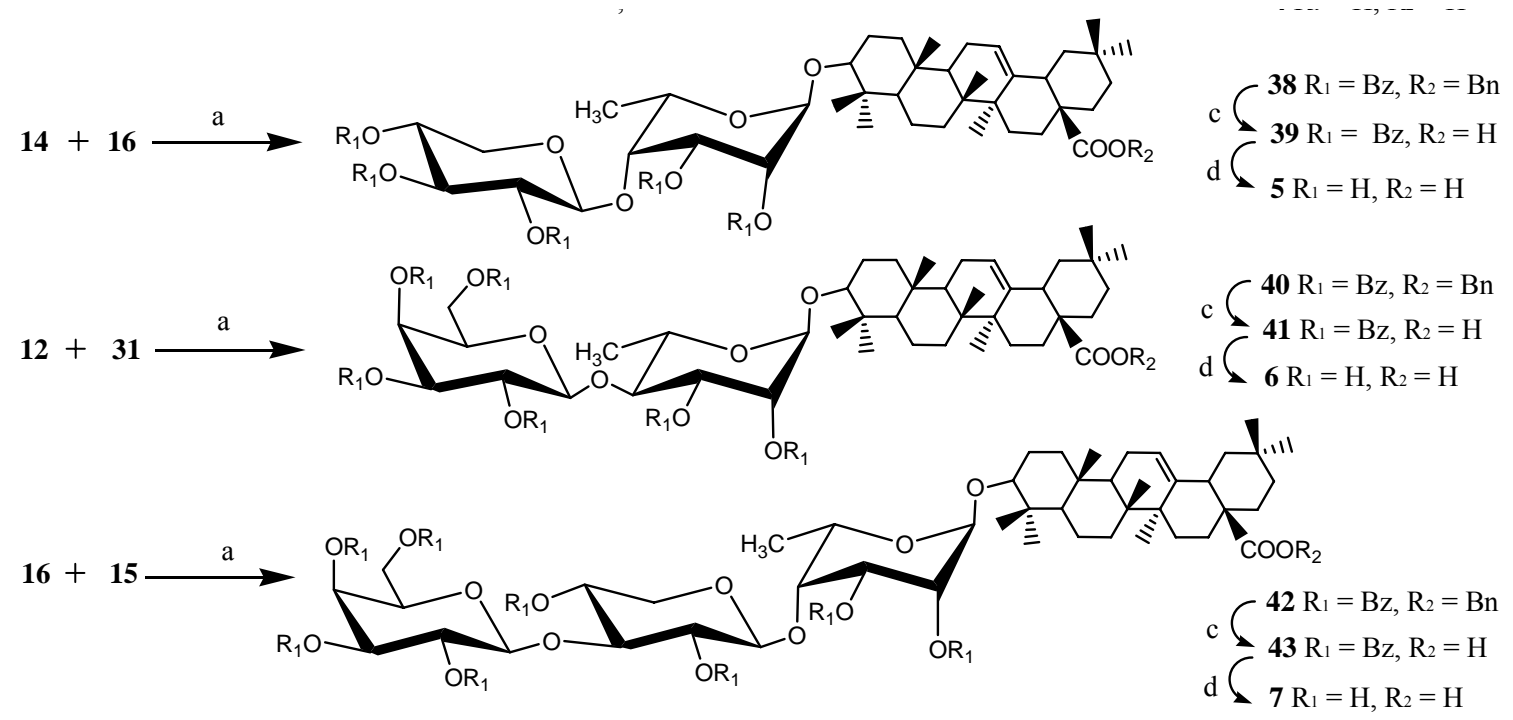

Reagents and conditions: (a) TMSOTf, $\mathrm{CH}_{2} \mathrm{Cl}_{2},-10{ }^{\circ} \mathrm{C}$ to r.t., $2 \mathrm{~h}, 88 \%$ for $\mathbf{3 0}, 90 \%$ for $\mathbf{3 4}, 86 \%$ for 36, $90 \%$ for 38, $89 \%$ for 40, 90\% for 42; (b) $\mathrm{PdCl}_{2}, \mathrm{MeOH}, 94 \%$ for 31; (c) $\mathrm{Pd}-\mathrm{C}, \mathrm{H}_{2}, 12 \mathrm{~h}$; (d) $\mathrm{MeOH}-\mathrm{MeONa}, 25 \mathrm{~h}, 86 \%$ for $\mathbf{1}, 81 \%$ for $\mathbf{2}, 84 \%$ for $3,87 \%$ for $\mathbf{4}, 71 \%$ for $\mathbf{5}, 68 \%$ for $\mathbf{6}, 75 \%$ for 7.

The 28-O-benzyl groups in 31, 16, 34, 36, 38, 40, 42 were removed with $\mathrm{Pd}-\mathrm{C}$ under $\mathrm{H}_{2}$ atmosphere, and then the $O$-benzoyl groups were cleaved with $\mathrm{MeOH}-\mathrm{MeONa}$ [19], furnishing the target compounds 1-7 in satisfactory yields, the structure of the target compounds were established by ${ }^{1} \mathrm{H}-\mathrm{NMR}$ and ${ }^{13} \mathrm{C}-\mathrm{NMR}$ spectroscopy. For example, the ${ }^{1} \mathrm{H}-\mathrm{NMR}$ spectrum of 7 showed characteristic signals such as $\delta 5.26,5.25,4.72 \mathrm{ppm}$ for three $\mathrm{H}-1$, and $\delta 1.28,1.00,0.99,0.95,0.90,0.83,0.78$ for the $\mathrm{CH}_{3}$ groups of oleanolic acid, the ${ }^{13} \mathrm{C}-\mathrm{NMR}$ spectrum showed peaks at $\delta 106.3,105.9,104.9 \mathrm{ppm}$ for three anomeric C-1s.

\subsection{Bioassay of Fungicidal Activities}

Fungicidal activities of the target compounds against Sclerotinia sclerotiorum (Lib.) de Bary, Rhizoctonia solani Kuhn, Botrytis cinerea Pers and Phytophthora parasitica Dast were evaluated using the mycelium growth rate test [20]. The diameter of the mycelia was measured and the inhibition rate was calculated according to formula (1):

$$
I=\frac{\bar{D}_{1}{ }^{2}-\bar{D}_{0}{ }^{2}}{\bar{D}_{1}{ }^{2}} \times 100 \%
$$

where $I$ is the inhibition rate, $\bar{D}_{1}$ is the average diameter of mycelia in the blank test, and $\bar{D}_{0}$ is the average diameter of mycelia in the presence of compounds 1-7: The inhibition rates of compounds 1-7 against the four fungi at $50 \mu \mathrm{g} / \mathrm{mL}$ are given in Table 1 . Compounds 1-7 exhibited more fungicidal activity against $R$. solani than the other fungi, compounds $\mathbf{1}$ and $\mathbf{2}$ are more active against $B$. cinerea and Phytophthora parasitica Dast than the other compounds. 
Table 1. Inhibition Rate of Compounds 1-7 against four Fungi.

\begin{tabular}{ccccc}
\hline & \multicolumn{4}{c}{ Inhibition rate (\%) } \\
\cline { 2 - 5 } Compd no. & S. sclerotiorum & R. solani & B. cinerea & $\begin{array}{c}\text { Phytophthora } \\
\text { parasitica Dast }\end{array}$ \\
\hline 1 & 71.90 & 96.05 & 75.41 & 79.21 \\
2 & 67.35 & 93.24 & 77.29 & 83.54 \\
3 & 78.27 & 95.29 & 68.42 & 67.24 \\
4 & 65.16 & 96.17 & 74.59 & 63.55 \\
5 & 73.47 & 93.86 & 71.73 & 52.57 \\
6 & 71.90 & 95.93 & 71.44 & 69.72 \\
7 & 71.10 & 88.48 & 67.17 & 70.06 \\
\hline
\end{tabular}

\section{Experimental}

\subsection{General methods}

Solvents were purified in the usual way. All commercially available reagents were used as received. All reactions were monitored by TLC analysis and TLC was performed on silica gel HF with detection by charring with $30 \%(\mathrm{v} / \mathrm{v}) \mathrm{H}_{2} \mathrm{SO}_{4}$ in $\mathrm{CH}_{3} \mathrm{OH}$ or by UV detection. Column chromatography was conducted by elution of a column $(8 \times 100,16 \times 240,18 \times 300,35 \times 400 \mathrm{~mm})$ of silica gel $(200-300$ mesh) with EtOAc-PE (b.p. 60-90 ${ }^{\circ} \mathrm{C}$ ) as the eluent. Air and moisture sensitive reactions were performed under dry $\mathrm{N}_{2}$ atmosphere. Optical rotations were recorded using a Perkin-Elmer 241 polarimeter. NMR spectra were recorded on a Varian XL-300 spectrometer with TMS as the internal standard. Elemental analysis was performed on a Yanaco CHN Corder MF-3 automatic elemental analyzer. Mass spectra were recorded with a VG PLATFORM mass spectrometer using the electronspray ionization (ESI) mode. Solutions were concentrated at a temperature $<60{ }^{\circ} \mathrm{C}$ under diminished pressure.

\subsection{Chemical synthesis}

p-Methoxyphenyl 4-O-allyl-2,3-O-isopropylidene- $\alpha$-L-rhamnopyranoside (18). Sodium hydride (2.3 g, $47.4 \mathrm{mmol})$ and allyl bromide $(3.6 \mathrm{~mL}, 41.1 \mathrm{mmol})$ were successively added to a soln. of compound 17 [14] (9.8 g, $31.6 \mathrm{mmol})$ in N,N-dimethylformamide $(50 \mathrm{~mL})$ which was cooled in an ice-salt bath. Then the reaction mixture was slowly allowed to reach room temperature and stirred for 20 min at the end of which time TLC (4:1 petroleum ether-EtOAc) indicated that the reaction was complete. The reaction mixture was diluted with EtOAc $(100 \mathrm{~mL})$, washed with ice-water, and dried $\left(\mathrm{Na}_{2} \mathrm{SO}_{4}\right)$. The soln was concentrated, and the residue was subjected to column chromatography (8:1 petroleum etherEtOAc) to give the desired product $18(10.5 \mathrm{~g}, 95 \%)$ as a foamy solid. $R_{\mathrm{f}}=0.68$ (4:1 petroleum etherEtOAc); $[\alpha]_{\mathrm{D}}^{25}-61.4\left(c\right.$ 0.5, $\left.\mathrm{CHCl}_{3}\right) ;{ }^{1} \mathrm{H}-\mathrm{NMR}\left(\mathrm{CDCl}_{3}\right): \delta 7.00-6.81(\mathrm{~m}, 4 \mathrm{H}, \mathrm{Bz}-\underline{\mathrm{H}}), 5.93(\mathrm{~m}, 1 \mathrm{H}$, $\left.\mathrm{CH}_{2}=\mathrm{C} \underline{\mathrm{H}}-\mathrm{CH}_{2} \mathrm{O}\right), 5.59(\mathrm{~s}, 1 \mathrm{H}, \mathrm{H}-1), 5.32-5.16(\mathrm{~m}, 2 \mathrm{H}), 4.40-4.32(\mathrm{~m}, 3 \mathrm{H}), 4.14(\mathrm{~m}, 1 \mathrm{H}), 3.83-3.77$ (m, $\left.4 \mathrm{H}, \mathrm{H}-5, \mathrm{OCH}_{3}\right), 3.21(\mathrm{~m}, 1 \mathrm{H}), 1.56\left(\mathrm{~s}, 3 \mathrm{H}, \underline{\mathrm{CH}}_{3}\right), 1.40$ (s, $\left.3 \mathrm{H}, \underline{\mathrm{CH}}_{3}\right), 1.23$ (d, $3 \mathrm{H}, J=6.3 \mathrm{~Hz}, \mathrm{H}-$ 6). Anal. Calcd. for $\mathrm{C}_{19} \mathrm{H}_{26} \mathrm{O}_{6}: \mathrm{C}, 65.13 ; \mathrm{H}, 7.48$; found: $\mathrm{C}, 65.29 ; \mathrm{H}, 7.63$. 
p-Methoxyphenyl 4-O-allyl-2,3-di-O-benzoyl- $\alpha$-L-rhamnopyranoside (19). Compound 18 (7.8 g, $22.3 \mathrm{mmol})$ was dissolved in $70 \%$ HOAc $(200 \mathrm{~mL})$ and stirred for $2 \mathrm{~h}$ at $75^{\circ} \mathrm{C}$, at the end of which time TLC (2:1 petroleum ether-EtOAc) indicated the completion of the reaction. The mixture was concentrated under reduced pressure and then coevaporated with toluene $(2 \times 40 \mathrm{~mL})$. To a soln of the residue $(7.3 \mathrm{~g}, 23.5 \mathrm{mmol})$ in pyridine $(60 \mathrm{~mL})$ was added benzoyl chloride $(8.2 \mathrm{~mL}, 70.5 \mathrm{mmol})$ dropwise. After stirring for $8 \mathrm{~h}$ at rt, TLC (3:1 petroleum ether-EtOAc) indicated that the reaction was complete. Methanol $(1 \mathrm{~mL})$ was added to quench the reaction and then water $(100 \mathrm{~mL})$ was added to the reaction mixture. The aq. soln. was extracted with EtOAc $(3 \times 200 \mathrm{~mL})$, the extract was washed with $1 \mathrm{M} \mathrm{HCl}$ and saturated aq. sodium bicarbonate, dried $\left(\mathrm{Na}_{2} \mathrm{SO}_{4}\right)$ and concentrated. The residue was passed through a short silica-gel column with 6:1 petroleum ether-EtOAc as the eluent to give 19 (10.2 $\mathrm{g}, 88 \%$ for two steps) as a foamy solid. $R_{\mathrm{f}}=0.42\left(4: 1\right.$ petroleum ether-EtOAc); $[\alpha]_{\mathrm{D}}^{25}+21.1(c$ 0.5, $\left.\mathrm{CHCl}_{3}\right) ;{ }^{1} \mathrm{H}-\mathrm{NMR}\left(\mathrm{CDCl}_{3}\right): \delta 8.07-7.34(\mathrm{~m}, 10 \mathrm{H}, \mathrm{Bz}-\underline{\mathrm{H}}), 7.08-6.83\left(\mathrm{~m}, 4 \mathrm{H}, \mathrm{MeOC}_{6} \underline{\mathrm{H}}_{4}\right), 5.87-$ 5.75 (m, $3 \mathrm{H}), 5.52$ (d, $1 \mathrm{H}, J=1.8 \mathrm{~Hz}, \mathrm{H}-1), 5.17$ (m, $1 \mathrm{H}), 5.08$ (m, $1 \mathrm{H}), 4.20-4.08$ (m, $3 \mathrm{H}), 3.78$ $3.71\left(\mathrm{~m}, 4 \mathrm{H}, \mathrm{H}-5, \mathrm{OCH}_{3}\right), 1.41$ (d, $\left.J=6.2 \mathrm{~Hz}, 3 \mathrm{H}, \mathrm{H}-6\right)$; Anal. Calcd. for $\mathrm{C}_{30} \mathrm{H}_{30} \mathrm{O}_{8}$ : C, 69.49; $\mathrm{H}$, 5.83; found: $\mathrm{C}, 69.55 ; \mathrm{H}, 5.58$;

4-O-Allyl-2,3-di-O-benzoyl- $\alpha$-L-rhamnopyranosyl trichloroacetimidate (10). To a soln. of 19 (10.0 g, $19.3 \mathrm{mmol})$ in $80 \% \mathrm{MeCN}(200 \mathrm{~mL})$ was added ceric ammonium nitrate (42.3 g, $77.2 \mathrm{mmol})$. The mixture was stirred for $20 \mathrm{~min}$ at $35^{\circ} \mathrm{C}$, at the end of which time TLC (4:1 petroleum ether-EtOAc) indicated that the reaction was complete. The solvents were evaporated in vacuo at $50{ }^{\circ} \mathrm{C}$ to give a residue, which was dissolved in $\mathrm{CH}_{2} \mathrm{Cl}_{2}$, and washed with water. The organic phase was dried $\left(\mathrm{Na}_{2} \mathrm{SO}_{4}\right)$ and concentrated. Purification by silica gel chromatography with 5:1 petroleum ether-EtOAc as the eluent afforded a foamy residue. The residue was dried under high vacuum for $2 \mathrm{~h}$, then was dissolved in dry $\mathrm{CH}_{2} \mathrm{Cl}_{2}(50 \mathrm{~mL})$, trichloroacetonitrile $(2.5 \mathrm{~mL}, 24.3 \mathrm{mmol})$ and 1,8-diazabicyclo[5.4.0] undecene (DBU) $(0.3 \mathrm{~mL}, 30 \mathrm{mmol})$ were added. The mixture was aged under the nitrogen atmosphere until completion (TLC, 4:1 petroleum ether-EtOAc). Concentration of the reaction mixture and purification of the residue by column chromatography $(5: 1$ petroleum etherEtOAc) gave 10 (7.6 g, 72\% for two steps) as a white foamy solid. $R_{\mathrm{f}}=0.67$ (4:1 petroleum etherEtOAc); $[\alpha]_{\mathrm{D}}^{25}+39.3\left(c \quad 0.5, \mathrm{CHCl}_{3}\right) ;{ }^{1} \mathrm{H}-\mathrm{NMR}\left(\mathrm{CDCl}_{3}\right): \delta 8.74(\mathrm{~s}, 1 \mathrm{H}, \mathrm{C}=\mathrm{NH}), 8.06-7.34(\mathrm{~m}, 10 \mathrm{H}$, Bz- $\underline{\mathrm{H}}$ ), 6.39 (d, $1 \mathrm{H}, J=1.9 \mathrm{~Hz}, \mathrm{H}-1), 5.85-5.71(\mathrm{~m}, 3 \mathrm{H}), 5.21-5.07$ (m, $2 \mathrm{H}), 4.22-4.15$ (m, $3 \mathrm{H}), 3.77$ (dd, $1 \mathrm{H}, J=9.6,9.6 \mathrm{~Hz}, \mathrm{H}-4), 1.48$ (d, $J=6.2 \mathrm{~Hz}, 3 \mathrm{H}, \mathrm{H}-6)$. Anal. Calcd. for $\mathrm{C}_{25} \mathrm{H}_{24} \mathrm{Cl}_{3} \mathrm{NO}_{7}$ : C, 53.93; H, 4.34; N, 2.52; found: C, 53.79; H, 4.23; N, 2.29.

p-Methoxyphenyl 4-O-allyl-2,3-O-isopropylidene-6-deoxy- $\alpha$-L-talopyranoside (21). Compound 20 (4.9 g, $15.8 \mathrm{mmol}$ ) was allylated under the same conditions as used for the preparation of 18 from 17, giving $21(5.1 \mathrm{~g}, 92 \%)$ as a foamy solid; $R_{\mathrm{f}}=0.73\left(4: 1\right.$ petroleum ether-EtOAc); $[\alpha]_{\mathrm{D}}^{25}-49.1(c 0.5$, $\left.\mathrm{CHCl}_{3}\right) ;{ }^{1} \mathrm{H}-\mathrm{NMR}\left(\mathrm{CDCl}_{3}\right): \delta 7.01-6.80(\mathrm{~m}, 4 \mathrm{H}, \mathrm{Bz}-\underline{\mathrm{H}}), 5.93\left(\mathrm{~m}, 1 \mathrm{H}, \mathrm{CH}_{2}=\mathrm{C}_{\underline{H}}-\mathrm{CH}_{2} \mathrm{O}\right), 5.56(\mathrm{~d}, 1 \mathrm{H}$, $J=1.5 \mathrm{~Hz}, \mathrm{H}-1)$, 5.29-5.17 (m, $2 \mathrm{H}), 4.49$ (m, $1 \mathrm{H}), 4.34-4.25$ (m, $2 \mathrm{H}), 4.09-4.00$ (m, $2 \mathrm{H}), 3.77$ (s, $\left.3 \mathrm{H}, \mathrm{OC}_{3}\right), 3.60$ (m, $\left.1 \mathrm{H}\right), 1.59$ (s, $\left.3 \mathrm{H}, \mathrm{CH}_{3}\right), 1.40$ (s, $\left.3 \mathrm{H}, \underline{\mathrm{C}}_{3}\right), 1.31$ (d, $\left.3 \mathrm{H}, J=6.6 \mathrm{~Hz}, \mathrm{H}-6\right)$. Anal. Calcd. for $\mathrm{C}_{19} \mathrm{H}_{26} \mathrm{O}_{6}$ : C, 65.13; H, 7.48; found: C, 65.25; H, 7.29. 
p-Methoxyphenyl 4-O-allyl-2,3-di-O-benzoyl-6-deoxy- $\alpha$-L-talopyranoside (22). Sequential de-Oisopropylidenation and then benzoylation of compound $21(7.8 \mathrm{~g}, 22.3 \mathrm{mmol})$ under the same conditions as those used for the preparation of 19 from 18, gave $22(9.3 \mathrm{~g}, 80 \%)$ as a foamy solid; $R_{\mathrm{f}}=0.67(3: 1 \text { petroleum ether-EtOAc); } \alpha]_{\mathrm{D}}^{25}-7.7\left(c \quad 0.5, \mathrm{CHCl}_{3}\right) ;{ }^{1} \mathrm{H}-\mathrm{NMR}\left(\mathrm{CDCl}_{3}\right): \delta 8.25-7.25(\mathrm{~m}$, $10 \mathrm{H}, \mathrm{Bz}-\underline{\mathrm{H}}), 7.07-6.82\left(\mathrm{~m}, 4 \mathrm{H}, \mathrm{MeOC}_{6} \underline{\mathrm{H}}_{4}\right), 5.93\left(\mathrm{~m}, 1 \mathrm{H}, \mathrm{CH}_{2}=\mathrm{C} \underline{\mathrm{H}}-\mathrm{CH}_{2} \mathrm{O}\right), 5.77$ (dd, $1 \mathrm{H}, J=3.49$, $3.34 \mathrm{~Hz}, \mathrm{H}-3), 5.68-5.67$ (m, $2 \mathrm{H}), 5.29-5.12$ (m, $2 \mathrm{H}), 4.33-4.27$ (m, $2 \mathrm{H}), 4.07$ (m, $1 \mathrm{H}), 3.83-3.76$ (m, $\left.4 \mathrm{H}, \mathrm{CH}_{2}=\mathrm{CH}-\underline{\mathrm{CH}}_{2} \mathrm{O}, \mathrm{OC}_{3}\right), 1.37$ (d, J =6.5 Hz, $3 \mathrm{H}, \mathrm{H}-6$ ); Anal. Calcd. for $\mathrm{C}_{30} \mathrm{H}_{30} \mathrm{O}_{8}: \mathrm{C}, 69.49$; $\mathrm{H}, 5.83$; found: $\mathrm{C}, 69.63 ; \mathrm{H}, 5.66$.

4-O-allyl-2,3-di-O-benzoyl-6-deoxy- $\alpha$-L-talopyranosyl trichloroacetimidate (11). Compound 22 (5.0 g, $9.7 \mathrm{mmol}$ ) was trichloroacetimidated under the same conditions as used for the preparation of $\mathbf{1 0}$ from 19, giving 11 (3.7 g, 70\% for two steps) as a foamy solid. $R_{\mathrm{f}}=0.70\left(4: 1\right.$ petroleum ether-EtOAc); $[\alpha]_{\mathrm{D}}^{25}$ $+6.14\left(c\right.$ 0.5, $\left.\mathrm{CHCl}_{3}\right) ;{ }^{1} \mathrm{H}-\mathrm{NMR}\left(\mathrm{CDCl}_{3}\right): \delta 8.74(\mathrm{~s}, 1 \mathrm{H}, \mathrm{C}=\mathrm{NH}), 8.24-7.32(\mathrm{~m}, 10 \mathrm{H}, \mathrm{Bz}-\underline{\mathrm{H}}), 6.48(\mathrm{~d}$, $J=1.4 \mathrm{~Hz}, 1 \mathrm{H}, \mathrm{H}-1), 5.90$ (m, $1 \mathrm{H}), 5.67-5.62$ (m, $2 \mathrm{H}), 5.18-5.09$ (m, $2 \mathrm{H}), 4.31-4.07$ (m, $2 \mathrm{H}), 3.87$ $(\mathrm{m}, 1 \mathrm{H}), 3.70(\mathrm{~m}, 1 \mathrm{H}), 1.44(\mathrm{~d}, J=6.5 \mathrm{~Hz}, 3 \mathrm{H}, \mathrm{H}-6)$. Anal. Calcd. for $\mathrm{C}_{25} \mathrm{H}_{24} \mathrm{Cl}_{3} \mathrm{NO}_{7}$ : C, 53.93; $\mathrm{H}$, 4.34; N, 2.52; found: C, 53.87; H, 4.15; N, 2.78.

Benzyl oleanolate 3-O-4-O-allyl-2,3-di-O-benzoyl-6-deoxy- $\alpha$-L-talopyranoside (23). Compound 11 (4.3 g, $7.8 \mathrm{mmol}), 9$ [10] (3.6 g, $6.4 \mathrm{mmol})$ and $4 \AA$ molecular sieves (1.0 g) were added to anhydrous redistilled $\mathrm{CH}_{2} \mathrm{Cl}_{2}(60 \mathrm{~mL})$. TMSOTf $(130 \mu \mathrm{L}, 0.7 \mathrm{mmol})$ was added dropwise at $-10{ }^{\circ} \mathrm{C}$ under nitrogen protection. The reaction mixture was allowed to raise to $\mathrm{rt}$ and stirred for $2 \mathrm{~h}$, and then quenched with $\mathrm{Et}_{3} \mathrm{~N}$ ( 2 drops). Filtration of the reaction mixture, concentration of the filtrate, followed by purification of the residue by column chromatography $(5: 1$ petroleum ether-EtOAc) provided 23 $(5.2 \mathrm{~g}, 85 \%) . R_{\mathrm{f}}=0.47\left(8: 1\right.$ petroleum ether-EtOAc). $[\alpha]_{\mathrm{D}}^{25}+39.3\left(c 0.5, \mathrm{CHCl}_{3}\right),{ }^{1} \mathrm{H}-\mathrm{NMR}\left(\mathrm{CDCl}_{3}\right): \delta$ 8.23-7.30 (m, $15 \mathrm{H}, \mathrm{Ar}-\underline{\mathrm{H}}), 5.88$ (m, $1 \mathrm{H}, \mathrm{CH}_{2}=\mathrm{C}_{-}-\mathrm{CH}_{2} \mathrm{O}$ ), 5.53 (dd, $\left.1 \mathrm{H}, J=3.4,3.5 \mathrm{~Hz}, \mathrm{H}-3^{\prime}\right), 5.42$ (m, $1 \mathrm{H}), 5.29$ (br s, $1 \mathrm{H}, \mathrm{H}-12), 5.23-5.02$ (m, $5 \mathrm{H}), 4.32-4.23$ (m, $2 \mathrm{H}), 4.03$ (m, $1 \mathrm{H}), 3.76$ (s, $1 \mathrm{H}$, $\mathrm{CH}_{2}=\mathrm{CH}-\underline{\mathrm{H}}_{2} \mathrm{O}$ ), 3.17 (dd, $\left.1 \mathrm{H}, J=5.1,10.7 \mathrm{~Hz}, \mathrm{H}-3\right), 2.90$ (dd, $\left.1 \mathrm{H}, J=3.8,13.7 \mathrm{~Hz}, \mathrm{H}-18\right), 1.35$ (d, $3 \mathrm{H}, J=6.5 \mathrm{~Hz}, \mathrm{H}-6$ '), 1.12, 1.01, 0.92, 0.91, 0.89, 0.83, $0.60\left(\mathrm{~s}, 7 \times 3 \mathrm{H}, \mathrm{CH}_{3}\right) ;{ }^{13} \mathrm{C}-\mathrm{NMR}\left(\mathrm{CDCl}_{3}\right): \delta$ 177.4, 166.3, $165.6(3 \mathrm{C}=\mathrm{O}), 143.7,136.4,135.0,133.1,133.0,130.3,130.1,129.7,129.7,128.4$, $128.4,128.4,128.4,128.4,128.2,128.2,128.0,128.0,128.0,127.9,122.5,116.7,100.6$ (C-1'), 89.3, 76.4, 74.3, 70.1, 69.0, 66.5, 65.9, 55.4, 47.6, 46.7, 45.9, 41.7, 41.4, 39.3, 39.0, 38.4, 36.7, 33.9, 33.1, $32.7,32.4,30.7,28.3,27.6,25.8,25.2,23.6,23.4,23.1,18.3,16.9,16.5,16.5,15.3$; Anal. Calcd. for $\mathrm{C}_{60} \mathrm{H}_{76} \mathrm{O}_{9}$ : C, 76.56; H, 8.14; found: C, 76.65; H, 8.31.

Benzyl oleanolate 3-O-2,3-di-O-benzoyl-6-deoxy- $\alpha$-L-talopyranoside (16). To a soln of compound 23 (5.0 g, $5.2 \mathrm{mmol})$ in $\mathrm{MeOH}-\mathrm{CH}_{2} \mathrm{Cl}_{2}=1 / 1(50 \mathrm{~mL})$ was added $\mathrm{PdCl}_{2}$ (304 mg, $\left.1.0 \mathrm{mmol}\right)$. The mixture was stirred for $12 \mathrm{~h}$, at the end of which time TLC (8:1 petroleum ether-EtOAc) indicated that the reaction was complete. The reaction mixture was diluted with dichloromethane $(100 \mathrm{~mL})$, washed with water and satd aq $\mathrm{Na}_{2} \mathrm{CO}_{3}$. The organic layer was concentrated, and the residue was passed through a short silica gel column with 8:1 petroleum ether-EtOAc as the eluent to give $\mathbf{1 6}(4.4 \mathrm{~g}, 92 \%) . R_{\mathrm{f}}=0.32$ (8:1 petroleum ether-EtOAc). $[\alpha]_{\mathrm{D}}^{25}+45.0\left(c 0.5, \mathrm{CHCl}_{3}\right),{ }^{1} \mathrm{H}-\mathrm{NMR}\left(\mathrm{CDCl}_{3}\right): \delta$ 8.07-7.26 (m, $15 \mathrm{H}, \mathrm{Ar}-$ $\underline{\mathrm{H}}$ ), 5.49-5.47 (m, 2 H), 5.29 (br s, 1 H, H-12), 5.07 (m, 3 H), 4.32-3.96 (m, 2 H, H-4', H-5'), 3.20 (dd, 
$1 \mathrm{H}, J=5.8,9.8 \mathrm{~Hz}, \mathrm{H}-3), 2.90$ (dd, $1 \mathrm{H}, J=4.4,13.9 \mathrm{~Hz}, \mathrm{H}-18), 2.55$ (d, $1 \mathrm{H}, J=11.1 \mathrm{~Hz}, \mathrm{OH}), 1.34$ $(\mathrm{d}, 3 \mathrm{H}, J=6.5 \mathrm{~Hz}, \mathrm{H}-6)$ ') 1.12, 1.02, 0.92, 0.92, 0.89, 0.85, $0.61\left(\mathrm{~s}, 7 \times 3 \mathrm{H}, \mathrm{CH}_{3}\right) ;{ }^{13} \mathrm{C}-\mathrm{NMR}$ $\left(\mathrm{CDCl}_{3}\right): \delta 177.4,165.5,165.5(3 \mathrm{C}=\mathrm{O}), 143.7,136.5,133.6,133.2,129.8,129.8,129.7,129.7,129.5$, 128.7, 128.7, 128.4, 128.4, 128.3, 128.0, 128.0, 127.9, 127.9, 126.8, 122.5, 100.4 (C-1'), 89.8, 70.6, 70.2, 68.9, 66.7, 65.9, 55.4, 47.6, 46.8, 45.9, 41.7, 41.4, 39.3, 39.0, 38.4, 36.7, 33.8, 33.1, 32.7, 32.4, 30.7, 28.3, 27.6, 25.9, 25.3, 23.6, 23.4, 23.1, 18.3, 16.9, 16.5, 16.2, 15.3; Anal. Calcd. for $\mathrm{C}_{57} \mathrm{H}_{72} \mathrm{O}_{9}$ : C, 75.97; H, 8.05; found: C, 75.83; H, 8.19.

\section{2,3,4,6-Tetra-O-benzoyl- $\beta$-D-galactopyranose-( $1 \rightarrow 3)$-1,2:5,6-di-O-isopropylidene- $\alpha$-D-glucofuranose}

(25). Compound 12 [11] (3.87 g, $5.2 \mathrm{mmol})$ and 24 [16] (1.24 g, $4.8 \mathrm{mmol})$ were coupled under the same conditions as that used for the preparation of 23 from $\mathbf{1 1}$ and $\mathbf{9}$, giving $25(3.5 \mathrm{~g}, 88 \%)$ as a foamy solid. $R_{\mathrm{f}}=0.16$ (4:1 petroleum ether-EtOAc); $[\alpha]_{\mathrm{D}}^{25}-61.4\left(c 0.5, \mathrm{CHCl}_{3}\right) ;{ }^{1} \mathrm{H}-\mathrm{NMR}\left(\mathrm{CDCl}_{3}\right): \delta$ 8.09-7.25 (m, 20 H, Bz- $\underline{\mathrm{H}}), 5.99$ (dd, $1 \mathrm{H}, J=0.8,3.3 \mathrm{~Hz}), 5.76$ (dd, $\left.1 \mathrm{H}, J=7.9,10.5 \mathrm{~Hz}, \mathrm{H}-2^{\prime}\right), 5.62$ (dd, $\left.1 \mathrm{H}, J=3.4,10.4 \mathrm{~Hz}, \mathrm{H}-3^{\prime}\right), 5.50$ (d, $\left.1 \mathrm{H}, J=3.6 \mathrm{~Hz}\right), 4.95$ (d, $\left.1 \mathrm{H}, J=7.9 \mathrm{~Hz}, \mathrm{H}-1^{\prime}\right), 4.67$ (dd, 1 $\mathrm{H}, J=6.3,11.1 \mathrm{~Hz}), 4.50-4.25(\mathrm{~m}, 6 \mathrm{H}), 4.16-4.03(\mathrm{~m}, 2 \mathrm{H}), 1.43,1.42,1.34,1.12\left(\mathrm{~s}, 4 \times 3 \mathrm{H}, \mathrm{CH}_{3}\right)$; ${ }^{13} \mathrm{C}-\mathrm{NMR}\left(\mathrm{CDCl}_{3}\right): \delta 166.0,165.5,165.5,164.9(4 \mathrm{C}=\mathrm{O}), 133.6,133.5,133.3,133.3,129.9,129.9$, $129.9,129.9,129.9,129.8,129.6,129.6,129.4,129.1,129.0,128.7,128.7,128.6,128.6,128.5,128.5$, 128.3, 128.3, 111.9, 108.6, 104.9, 100.6 (2 × C-1), 82.9, 81.8, 80.5, 77.2, 73.1, 71.8, 71.5, 69.9, 68.0, 66.3, 61.9, 26.7, 26.6, 25.9, 25.3; Anal. Calcd. for $\mathrm{C}_{46} \mathrm{H}_{46} \mathrm{O}_{15}$ : C, 65.86; H, 5.53; found: C, 65.72; $\mathrm{H}, 5.75$.

\section{2,3,4,6-Tetra-O-benzoyl- $\beta$-D-galactopyranose-(1 $\rightarrow 3)$-1,2-O-isopropylidene- $\alpha$-D-glucofuranose}

(26).

The compound $25(3.0 \mathrm{~g})$ was dissolved in $60 \% \mathrm{HOAc}(100 \mathrm{~mL})$ and stirred for $6 \mathrm{~h}$ at $25^{\circ} \mathrm{C}$, at the end of which time TLC $(2: 1$ petroleum ether-EtOAc) indicated the completion of the reaction. The mixture was concentrated under reduced pressure and then co evaporated with toluene $(2 \times 40 \mathrm{~mL})$. The residue was passed through a short silica-gel column with 3:1 petroleum ether-EtOAc as the eluent to give $26(2.4 \mathrm{~g}, 83 \%)$ as a foamy solid. $R_{\mathrm{f}}=0.68\left(1: 1\right.$ petroleum ether-EtOAc); $[\alpha]_{\mathrm{D}}^{25}+98.2(c 1.0$, $\left.\mathrm{CHCl}_{3}\right) ;{ }^{1} \mathrm{H}-\mathrm{NMR}\left(\mathrm{CDCl}_{3}\right): \delta 8.08-7.23(\mathrm{~m}, 20 \mathrm{H}, \mathrm{Bz}-\underline{\mathrm{H}}), 6.01(\mathrm{~d}, 1 \mathrm{H}, J=2.5 \mathrm{~Hz}), 5.79(\mathrm{dd}, 1 \mathrm{H}$, $\left.J=8.0,10.5 \mathrm{~Hz}, \mathrm{H}-2^{\prime}\right), 5.61$ (dd, $\left.1 \mathrm{H}, J=3.4,10.5 \mathrm{~Hz}, \mathrm{H}-3^{\prime}\right), 5.53(\mathrm{~d}, 1 \mathrm{H}, J=3.7 \mathrm{~Hz}), 4.98$ (d, $1 \mathrm{H}$, $J=7.9 \mathrm{~Hz}, \mathrm{H}-1$ '), 4.57 (d, $2 \mathrm{H}, J=6.1 \mathrm{~Hz}), 4.50-4.29$ (m, $3 \mathrm{H}), 4.23$ (d, $1 \mathrm{H}, J=3.7 \mathrm{~Hz}), 4.19-4.07$ (m, $3 \mathrm{H}), 3.92-3.85(\mathrm{~m}, 1 \mathrm{H}), 3.69(\mathrm{dd}, 1 \mathrm{H}, J=5.7,11.5 \mathrm{~Hz}, \mathrm{H}-18), 1.42,1.06\left(\mathrm{~s}, 2 \times 3 \mathrm{H}, \mathrm{CH}_{3}\right) ;{ }^{13} \mathrm{C}-$ NMR $\left(\mathrm{CDCl}_{3}\right): \delta 166.1,165.5,165.5,164.8(4 \mathrm{C}=\mathrm{O}), 133.8,133.6,133.4,133.4,133.3,130.0,129.9$, $129.8,129.8,129.8,129.6,129.1,129.0,129.0,128.8,128.8,128.7,128.7,128.6,128.6,128.5,128.3$, 128.3, 112.2, 105.2, 101.9 (2 × C-1), 83.6, 83.2, 80.0, 77.2, 72.4, 71.3, 69.5, 68.7, 68.0, 64.4, 62.2, 26.7, 26.2; Anal. Calcd. for $\mathrm{C}_{43} \mathrm{H}_{42} \mathrm{O}_{15}$ : C, 64.66; H, 5.30; found: C, 64.49; H, 5.38.

\section{2,3,4,6-Tetra-O-benzoyl- $\beta$-D-galactopyranose- $(1 \rightarrow 3)$-5-aldehyde-1,2-O-isopropylidene- $\alpha$-D-gluco-} furanose (27). To a vigorously stirred suspension of silicagel-supported $\mathrm{NaIO}_{4}$ reagent which was prepared as the reported method [17] $(2.0 \mathrm{~g})$ in $\mathrm{CH}_{2} \mathrm{Cl}_{2}(5 \mathrm{~mL})$ was added a soln of the compound 26 $(0.8 \mathrm{~g}, 1 \mathrm{mmol})$ in $\mathrm{CH}_{2} \mathrm{Cl}_{2}(5 \mathrm{~mL})$. The mixture was stirred at $\mathrm{rt}$ for $25 \mathrm{~min}$, and TLC (2:1 petroleum ether-EtOAc) indicated that the reaction was complete. The mixture was filtered, and the silica gel was thoroughly washed with $\mathrm{CHCl}_{3}$. Purification by silica gel chromatography with $2: 1$ petroleum ether- 
EtOAc as the eluent afforded $27(0.7 \mathrm{~g}, 88 \%)$ as a foamy solid. $R_{\mathrm{f}}=0.41(2: 1$ petroleum ether-EtOAc); $[\alpha]_{\mathrm{D}}^{25}+70.2\left(c 0.5, \mathrm{CHCl}_{3}\right) ;{ }^{1} \mathrm{H}-\mathrm{NMR}\left(\mathrm{CDCl}_{3}\right): \delta 9.68(\mathrm{~d}, 1 \mathrm{H}, J=1.5 \mathrm{~Hz}, \mathrm{CHO}), 8.08-7.25(\mathrm{~m}, 20 \mathrm{H}$, Bz- $\underline{\mathrm{H}}), 5.97$ (dd, $1 \mathrm{H}, J=0.9,3.4 \mathrm{~Hz}), 5.74-5.59$ (m, $3 \mathrm{H}), 4.89$ (d, $\left.1 \mathrm{H}, J=7.8 \mathrm{~Hz}, \mathrm{H}-1^{\prime}\right), 4.70-4.54$ $(\mathrm{m}, 3 \mathrm{H}), 4.48-4.30(\mathrm{~m}, 3 \mathrm{H}), 1.44,1.18\left(\mathrm{~s}, 2 \times 3 \mathrm{H}, \mathrm{CH}_{3}\right) ;{ }^{13} \mathrm{C}-\mathrm{NMR}\left(\mathrm{CDCl}_{3}\right): \delta 197.9,166.0,165.7$, $165.5,164.8(5 \mathrm{C}=\mathrm{O}), 133.7,133.6,133.3,133.3,130.3,130.0,130.0,129.9,129.8,129.8,129.8$, 129.6, 129.6, 129.4, 129.2, 129.1, 129.0, 128.9, 128.8, 128.7, 128.7, 128.6, 128.5, 128.3, 112.8, 105.7, $100.6(2 \times \mathrm{C}-1), 83.9,83.0,82.9,77.2,71.8,71.5,69.6,67.9,61.9,26.6,26.1$; Anal. Calcd. for $\mathrm{C}_{43} \mathrm{H}_{40} \mathrm{O}_{14}$ : C, 66.15; H, 5.16; found: C, 66.34; H, 5.25.

2,3,4,6-Tetra-O-benzoyl- $\beta$-D-galactopyranose-( $1 \rightarrow 3)$-1,2-O-isopropylidene- $\beta$ - $D$-xylose $(\mathbf{2 8})$. To a soln of $27(1.4 \mathrm{~g}, 1.8 \mathrm{mmol})$ in $7: 3 \mathrm{EtOAc}-\mathrm{H}_{2} \mathrm{O}(50 \mathrm{~mL})$ at $0{ }^{\circ} \mathrm{C}$ was added $\mathrm{NaBH}_{4}(109 \mathrm{mg}, 2.7 \mathrm{mmol})$. The mixture was stirred at $0{ }^{\circ} \mathrm{C}$ for $15 \mathrm{~min}$, and TLC $(3: 1$ petroleum ether-EtOAc) indicated that the reaction was complete. The aq. soln. was extracted with EtOAc $(3 \times 100 \mathrm{~mL})$, the extract was washed with $1 \mathrm{M} \mathrm{HCl}$ and saturated aq sodium bicarbonate, dried $\left(\mathrm{Na}_{2} \mathrm{SO}_{4}\right)$ and concentrated. Purification by silica gel chromatography with 5:1 petroleum ether-EtOAc as the eluent afforded $\mathbf{2 8}(1.3 \mathrm{~g}, 96 \%)$ as a foamy solid. $R_{\mathrm{f}}=0.29$ (3:2 petroleum ether-EtOAc); $[\alpha]_{\mathrm{D}}^{25}+184.2\left(c 1.0, \mathrm{CHCl}_{3}\right) ;{ }^{1} \mathrm{H}-\mathrm{NMR}\left(\mathrm{CDCl}_{3}\right): \delta$ 8.08-7.26 (m, $20 \mathrm{H}, \mathrm{Bz}-\underline{\mathrm{H}}), 6.00(\mathrm{dd}, 1 \mathrm{H}, J=0.8,3.3 \mathrm{~Hz}), 5.80-5.55(\mathrm{~m}, 3 \mathrm{H}), 4.96(\mathrm{~d}, 1 \mathrm{H}$, $\left.J=7.9 \mathrm{~Hz}, \mathrm{H}-1^{\prime}\right), 4.70-4.30$ (m, $\left.6 \mathrm{H}\right), 4.16-3.91$ (m, $\left.2 \mathrm{H}\right), 2.56$ (dd, $\left.1 \mathrm{H}, J=6.7,6.7 \mathrm{~Hz}\right), 1.44,1.12$ (s, $\left.2 \times 3 \mathrm{H}, \mathrm{CH}_{3}\right) ;{ }^{13} \mathrm{C}-\mathrm{NMR}\left(\mathrm{CDCl}_{3}\right): \delta 166.1,165.5,165.5,164.9(4 \mathrm{C}=\mathrm{O}), 133.8,133.6,133.4,, 133.4$, $130.1,130.1,130.0,130.0,129.8,129.8,129.7,129.6,129.2,129.0,128.9,128.8,128.7,128.7,128.7$, 128.6, 128.5, 128.5, 128.3, 112.1, 104.9, 101.3 (2 × C-1), 83.6, 82.8, 79.8, 77.2, 72.1, 71.4, 69.6, 68.0, 62.2, 59.9, 26.9, 26.1; Anal. Calcd. for $\mathrm{C}_{42} \mathrm{H}_{40} \mathrm{O}_{14}$ : C, 65.62; H, 5.24; found: $\mathrm{C}, 65.35$; H, 5.37 .

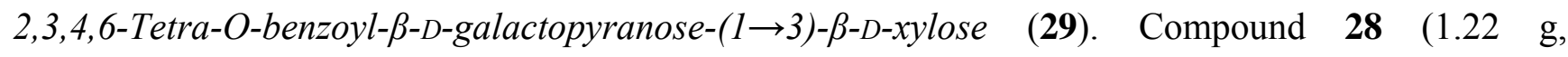
$1.6 \mathrm{mmol})$ was dissolved in $4 \%$ aq $\mathrm{H}_{2} \mathrm{SO}_{4}(100 \mathrm{~mL})$ and then refluxed for $4 \mathrm{~h}$. TLC (1:1 petroleum ether-EtOAc) indicated that the reaction was complete. The resulting soln. was cooled down to room temperature and extracted three times with EtOAc. The extract was washed with saturated aq. sodium bicarbonate, dried $\left(\mathrm{Na}_{2} \mathrm{SO}_{4}\right)$ and concentrated. Purification by silica gel chromatography with $2: 1$ petroleum ether-EtOAc as the eluent afforded $29(0.9 \mathrm{~g}, 85 \%)$ as a foamy solid. $R_{\mathrm{f}}=0.31(1: 1$ petroleum ether-EtOAc); $[\alpha]_{\mathrm{D}}^{25}+331.6\left(\mathrm{c} 1.0, \mathrm{CHCl}_{3}\right) ;{ }^{1} \mathrm{H}-\mathrm{NMR}\left(\mathrm{CDCl}_{3}\right): \delta$ 8.10-7.25 (m, $20 \mathrm{H}, \mathrm{Bz}-$ $\underline{\mathrm{H}}), 6.01(\mathrm{~d}, 1 \mathrm{H}, J=3.3 \mathrm{~Hz}), 5.85(\mathrm{~m}, 1 \mathrm{H}), 5.65(\mathrm{~m}, 1 \mathrm{H}), 5.07-5.02(\mathrm{~m}, 2 \mathrm{H}), 4.58-4.40(\mathrm{~m}, 3 \mathrm{H})$, 3.79-3.74 (m, $3 \mathrm{H}), 3.50(\mathrm{~m}, 1 \mathrm{H}), 3.27(\mathrm{~m}, 1 \mathrm{H}) ;{ }^{13} \mathrm{C}-\mathrm{NMR}\left(\mathrm{CDCl}_{3}\right): \delta 166.1,165.6,165.5,165.5(4$ $\mathrm{C}=\mathrm{O}), 133.7,133.5,133.4,133.4,130.0,130.0,129.8,129.7,129.1,129.0,128.8,128.8,128.7,128.6$, 128.5, 128.5, 128.4, 128.3, 102.8, 102.6 (2 × C-1), 97.3, 92.4, 88.2, 85.7, 77.2, 73.4, 72.0, 71.5, 70.7, 70.0, 69.9, 68.2, 68.1, 62.4, 62.0; Anal. Calcd. for $\mathrm{C}_{39} \mathrm{H}_{36} \mathrm{O}_{14}: \mathrm{C}, 64.28$; H, 4.98; found: C, 64.39; $\mathrm{H}, 4.83$.

2,3,4,6-Tetra-O-benzoyl- $\beta$-D-galactopyranose- $(1 \rightarrow 3)$-2,4-di-O-benzoyl- $\beta$-D-xylopyranosyl trichloroacetimidate (15). Compound $29(3.5 \mathrm{~g}, 4.8 \mathrm{mmol})$ was benzoylated under the same conditions as used for the preparation of 19. Then the resultant residue was dissolved in $2{\mathrm{M} \mathrm{MeOH}-\mathrm{NH}_{3}}(200 \mathrm{~mL})$ and stirred at $35{ }^{\circ} \mathrm{C}$ at the end of which time TLC (3:1 petroleum ether-EtOAc) indicated that the reaction 
was complete. The solvents were evaporated in vacuo at $50{ }^{\circ} \mathrm{C}$ to give a residue, which was dissolved in $\mathrm{CH}_{2} \mathrm{Cl}_{2}$, and washed with water. The organic phase was dried $\left(\mathrm{Na}_{2} \mathrm{SO}_{4}\right)$ and concentrated. Purification by silica gel chromatography with 5:1 petroleum ether-EtOAc as the eluent afforded a foamy residue. The residue was trichloroacetimidated under the same conditions as used for the preparation of 10 from 19, giving 15 (3.5 g, 68\% for three steps) as a white foamy solid. $R_{\mathrm{f}}=0.42(3: 1$ petroleum ether-EtOAc); $[\alpha]_{\mathrm{D}}^{25}+18.4\left(c 0.5, \mathrm{CHCl}_{3}\right) ;{ }^{1} \mathrm{H}-\mathrm{NMR}\left(\mathrm{CDCl}_{3}\right): \delta 8.52(\mathrm{~s}, 1 \mathrm{H}, \mathrm{C}=\mathrm{N} \underline{\mathrm{H}}), 8.18$ $7.08(\mathrm{~m}, 30 \mathrm{H}, \mathrm{Bz}-\underline{\mathrm{H}}$ ), 6.54 (d, $1 \mathrm{H}, J=3.5 \mathrm{~Hz}), 5.87$ (d, $1 \mathrm{H}, J=3.3 \mathrm{~Hz}), 5.66$ (dd, $1 \mathrm{H}, J=7.9,10.4$ Hz), 5.48-5.37 (m, 2 H), 5.27 (dd, $1 \mathrm{H}, J=3.5,9.7 \mathrm{~Hz}), 5.14$ (d, $1 \mathrm{H}, J=7.9 \mathrm{~Hz}, \mathrm{H}-1$ '), 4.67 (dd, 1 H, $J=9.4,9.4 \mathrm{~Hz}), 4.41-4.18(\mathrm{~m}, 4 \mathrm{H}), 3.95(\mathrm{dd}, 1 \mathrm{H}, J=10.9,11.0 \mathrm{~Hz})$. Anal. Calcd. for $\mathrm{C}_{55} \mathrm{H}_{44} \mathrm{Cl}_{3} \mathrm{NO}_{16}$ : C, 61.09; H, 4.10; N, 1.30; found: $\mathrm{C}, 61.34 ; \mathrm{H}, 4.27 ; \mathrm{N}, 1.49$.

Benzyl oleanolate 3-O-4-O-allyl-2,3-di-O-benzoyl- $\alpha$-L-rhamnopyranoside (30). Compound 10 (4.3 g, $7.8 \mathrm{mmol}$ ) and 9 [10] (3.6 g, $6.4 \mathrm{mmol})$ were coupled under the same conditions as used for the preparation of 23 from 11 and $\mathbf{9}$, giving $30(5.4 \mathrm{~g}, 88 \%)$ as a foamy solid. $R_{\mathrm{f}}=0.45$ (8:1 petroleum ether-EtOAc); $[\alpha]_{\mathrm{D}}^{25}+73.7\left(c\right.$ 0.5, $\left.\mathrm{CHCl}_{3}\right) ;{ }^{1} \mathrm{H}-\mathrm{NMR}\left(\mathrm{CDCl}_{3}\right): \delta 8.06-7.31(\mathrm{~m}, 15 \mathrm{H}, \mathrm{Ar}-\underline{\mathrm{H}}), 5.81(\mathrm{~m}$, $1 \mathrm{H}, \mathrm{CH}_{2}=\mathrm{CH}_{-}-\mathrm{CH}_{2} \mathrm{O}$ ), 5.66 (dd, $\left.1 \mathrm{H}, J=3.2,9.5 \mathrm{~Hz}, \mathrm{H}-3^{\prime}\right), 5.57$ (dd, $\left.1 \mathrm{H}, J=1.7,3.2 \mathrm{~Hz}, \mathrm{H}-2^{\prime}\right), 5.29$ (br s, $1 \mathrm{H}, \mathrm{H}-12$ ), 5.20-5.03 (m, $4 \mathrm{H}, \mathrm{PhC} \underline{\mathrm{H}} 2, \mathrm{C}_{2}=\mathrm{CH}-\mathrm{CH}_{2} \mathrm{O}$ ), 4.97 (d, $1 \mathrm{H}, J_{1,2}=1.7 \mathrm{~Hz}, \mathrm{H}-1$ '), $4.22-$ 4.04 (m, $3 \mathrm{H}$ ), 3.67 (dd, $1 \mathrm{H}, J=9.5,9.5 \mathrm{~Hz}, \mathrm{H}-4$ '), 3.15 (dd, $1 \mathrm{H}, J=6.3,9.8 \mathrm{~Hz}, \mathrm{H}-3$ ), 2.91 (dd, $1 \mathrm{H}$, $J=4.1,13.4 \mathrm{~Hz}, \mathrm{H}-18), 1.40$ (d, $3 \mathrm{H}, J=6.2 \mathrm{~Hz}, \mathrm{H}-6$ ) $), 1.12,1.02,0.92,0.92,0.89,0.88,0.61$ (s, $\left.7 \times 3 \mathrm{H}, \mathrm{CH}_{3}\right) ;{ }^{13} \mathrm{C}-\mathrm{NMR}\left(\mathrm{CDCl}_{3}\right): \delta 177.4,165.5,165.3(3 \mathrm{C}=\mathrm{O}), 143.7,136.4,134.5,133.2,132.9$, 130.0, 130.0, 129.9, 129.7, 129.5, 129.5, 128.4, 128.4, 128.4, 128.4, 128.3, 128.0, 128.0, 127.9, 122.5, 117.2, 99.6 (C-1'), 89.7, 79.1, 77.2, 74.0, 72.5, 71.5, 67.8, 65.9, 55.4, 47.6, 45.9, 41.7, 41.4, 39.3, 39.0, 38.4, 36.7, 33.9, 33.1, 32.7, 32.4, 30.7, 28.3, 27.6, 25.9, 25.3, 23.6, 23.4, 23.1, 18.3, 18.0, 16.9, 16.5, 15.3; Anal. Calcd. for $\mathrm{C}_{60} \mathrm{H}_{76} \mathrm{O}_{9}$ : C, 76.56; H, 8.14; found: C, 76.73; H, 8.43.

Benzyl oleanolate 3-O-2,3-di-O-benzoyl- $\alpha$-L-rhamnopyranoside (31). Compound 30 (5.0 g, 5.2 mmol) was deallylated under the same conditions as that used for the preparation of $\mathbf{1 6}$ from 23, giving 31 $(4.5 \mathrm{~g}, 94 \%)$ as a foamy solid; $R_{\mathrm{f}}=0.16\left(8: 1\right.$ petroleum ether-EtOAc); $[\alpha]_{\mathrm{D}}^{25}+29.5\left(c 1.0, \mathrm{CHCl}_{3}\right) ;{ }^{1} \mathrm{H}-$ NMR $\left(\mathrm{CDCl}_{3}\right): \delta$ 8.09-7.26 (m, 15 H, Ar- $\left.\underline{\mathrm{H}}\right), 5.57-5.48$ (m, $\left.2 \mathrm{H}\right), 5.29$ (br s, $\left.1 \mathrm{H}, \mathrm{H}-12\right), 5.07$ (dd, $2 \mathrm{H}$, $\left.J=12.6,17.1 \mathrm{~Hz}, \mathrm{PhCH}_{2}\right), 5.00(\mathrm{~d}, 1 \mathrm{H}, J=1.6 \mathrm{~Hz}, \mathrm{H}-1$ ), $4.06-3.87$ (m, $2 \mathrm{H}), 3.18$ (dd, $1 \mathrm{H}$, $J=2.9,13.0 \mathrm{~Hz}, \mathrm{H}-3), 2.90$ (dd, $1 \mathrm{H}, J=4.4,14.2 \mathrm{~Hz}, \mathrm{H}-18), 2.49$ (d, $1 \mathrm{H}, J=5.1 \mathrm{~Hz}, \mathrm{O} \underline{\mathrm{H}}), 1.41$ (d, $3 \mathrm{H}, J=6.1 \mathrm{~Hz}, \mathrm{H}-6$ '), 1.12, 1.01, 0.92, 0.92, 0.89, 0.87, $0.61\left(\mathrm{~s}, 7 \times 3 \mathrm{H}, \mathrm{CH}_{3}\right) ;{ }^{13} \mathrm{C}-\mathrm{NMR}\left(\mathrm{CDCl}_{3}\right): \delta$ 177.4, 166.8, 165.6 (3 C=O), 143.6, 136.4, 133.3, 133.2, 129.7, 129.7, 129.7, 129.7, 129.5, 129.4, $128.4,128.4,128.3,128.3,127.9,127.9,127.9,127.8,126.8,122.4,99.7$ (C-1'), 89.7, 73.4, 72.1, 71.3, 68.7, 65.9, 55.4, 47.5, 46.7, 45.8, 41.6, 41.4, 39.3, 38.9, 38.4, 36.7, 33.8, 33.0, 32.7, 32.3, 30.6, 28.3, 27.6, 25.8, 25.3, 23.6, 23.4, 23.0, 18.2, 17.5, 16.8, 16.5, 15.3; Anal. Calcd. for $\mathrm{C}_{57} \mathrm{H}_{72} \mathrm{O}_{9}$ : C, 75.97; $\mathrm{H}$, 8.05; found: $\mathrm{C}, 75.81 ; \mathrm{H}, 8.29$.

Oleanolic acid 3-O- $\alpha$-L-rhamnopyranoside (1). A suspension of 31 (1.3 g, $1.4 \mathrm{mmol})$ and 10\% Pd-C $(1.5 \mathrm{~g})$ in EtOAc $(30 \mathrm{~mL})$ was refluxed and bubbled up with $\mathrm{H}_{2}(20 \mathrm{~mL} / \mathrm{min})$. When TLC (2:1, petroleum-EtOAc) showed that the reaction had completed, $\mathrm{Pd}-\mathrm{C}$ was removed through filtration and the filtrate was concentrated to dryness. The resulted amorphous solid was dissolved in $\operatorname{dry} \mathrm{CH}_{2} \mathrm{Cl}_{2}-$ 
$\mathrm{MeOH}(1: 2,30 \mathrm{~mL})$, to which a newly prepared $\mathrm{NaOMe} / \mathrm{MeOH}(1.0 \mathrm{~mol} / \mathrm{L}, 20 \mathrm{~mL})$ was added. The soln was stirred at $\mathrm{rt}$ for $2 \mathrm{~h}$ and then neutralized with Dowex $\mathrm{H}^{+}$resin to $\mathrm{pH} 7$ and filtered. The filtrate was concentrated and subjected to a flash column chromatography $\left(\mathrm{CHCl}_{3}-\mathrm{MeOH}-\mathrm{H}_{2} \mathrm{O}\right.$ 7:3:1, organic layer) to give $\mathbf{1}$ [13] (737 $\mathrm{mg}, 86 \%$ for two steps) as a white powder.

Oleanolic acid 3-O-6-deoxy- $\alpha$-L-talopyranoside (2). Compound 2 was prepared from 16 by the same procedure as for 1. Yield: 81\%; white powder, m.p. $288-290{ }^{\circ} \mathrm{C}, R_{\mathrm{f}}=0.29\left(10: 1: 0.1 \mathrm{CHCl}_{3}-\mathrm{MeOH}-\right.$ $\left.\mathrm{H}_{2} \mathrm{O}\right) ;[\alpha]_{\mathrm{D}}^{25}+6.1(c$ 0.5, MeOH)$){ }^{1} \mathrm{H}-\mathrm{NMR}$ (pyridine- $\left.\mathrm{d}_{5}\right): \delta 5.47$ (br s, $\left.1 \mathrm{H}, \mathrm{H}-12\right), 5.31(\mathrm{~d}, 1 \mathrm{H}$, $\left.J=1.3 \mathrm{~Hz}, \mathrm{H}-1^{\prime}\right), 4.85$ (dd, $\left.1 \mathrm{H}, J=1.5,3.0 \mathrm{~Hz}, \mathrm{H}-2^{\prime}\right), 4.25-4.21(\mathrm{~m}, 2 \mathrm{H}), 4.06$ (d, $\left.1 \mathrm{H}, J=1.4 \mathrm{~Hz}\right)$, 3.29 (dd, $1 \mathrm{H}, J=4.0,13.7 \mathrm{~Hz}, \mathrm{H}-3), 3.13$ (dd, $1 \mathrm{H}, J=4.3,11.5 \mathrm{~Hz}, \mathrm{H}-18), 1.54$ (d, $3 \mathrm{H}, J=6.5 \mathrm{~Hz}$, $\left.\mathrm{H}^{6} 6^{\prime}\right), 1.28,1.00,0.99,0.95,0.90,0.85,0.80\left(\mathrm{~s}, 7 \times 3 \mathrm{H}, \mathrm{CH}_{3}\right) ;{ }^{13} \mathrm{C}-\mathrm{NMR}$ (pyridine- $\left.\mathrm{d}_{5}\right): \delta 180.2,144.9$, 122.6, 104.9 (C-1'), 88.6, 74.3, 72.4, 67.8, 67.5, 55.7, 48.1, 46.7, 46.6, 42.2, 42.1, 39.8, 39.2, 38.6, 37.1, 34.3, 33.4, 33.3, 33.2, 31.0, 28.4, 28.3, 26.2, 25.8, 23.9, 23.9, 23.8, 18.7, 17.5, 17.4, 16.8, 15.5; HRESIMS: $m / z$ calcd. for $\mathrm{C}_{36} \mathrm{H}_{58} \mathrm{O}_{7} \mathrm{Na}\left[\mathrm{M}+\mathrm{Na}^{+}\right.$]: 625.4080 ; found: $m / z 625.4059$.

Benzyl oleanolate 3-O-2,3,4,6-tetra-O-benzoyl- $\beta$-D-galactopyranoside (34). Compound 12 (0.56 g, $0.8 \mathrm{mmol})$ and 9 [15] (0.6 g, $0.7 \mathrm{mmol})$ were coupled under the same conditions as that used for the preparation of 23 from 11 and 9, giving 34 [13] (0.9 g, 90\%) as a foamy solid.

Oleanolic acid 3-O- $\beta$-D-galactopyranoside (3). Compound 3 [13] was prepared from 34 by the same procedure as for 1 . Yield: $84 \%$; white powder.

Benzyl oleanolate 3-O-2,3,4,6-tetra-O-acetyl- $\alpha$-D-mannopyranoside (36). Compound 13 [12] (1.5 g, $3.0 \mathrm{mmol})$ and 9 [10] (1.4 g, $2.5 \mathrm{mmol})$ were coupled under the same conditions as used for the preparation of 23 from 11 and 9 , giving $36(1.9 \mathrm{~g}, 86 \%)$ as a foamy solid. $R_{\mathrm{f}}=0.16$ (6:1 petroleum ether-EtOAc); $[\alpha]_{\mathrm{D}}^{25}+70.6\left(c\right.$ 0.5, $\left.\mathrm{CHCl}_{3}\right) ;{ }^{1} \mathrm{H}-\mathrm{NMR}\left(\mathrm{CDCl}_{3}\right): \delta$ 7.47-7.27 (m, $\left.5 \mathrm{H}, \mathrm{Bn}-\underline{\mathrm{H}}\right)$, 5.35-5.24 $(\mathrm{m}, 3 \mathrm{H}), 5.16-5.06(\mathrm{~m}, 3 \mathrm{H}), 4.97(\mathrm{~d}, 1 \mathrm{H}, J=1.7 \mathrm{~Hz}, \mathrm{H}-1$ '), 4.25 (dd, $1 \mathrm{H}, J=5.7,12.5 \mathrm{~Hz}, \mathrm{H}-3)$, 4.15-4.10 (m, $2 \mathrm{H}), 3.21$ (dd, $1 \mathrm{H}, J=4.0,11.3 \mathrm{~Hz}, \mathrm{H}-3), 2.90$ (dd, $1 \mathrm{H}, J=4.0,13.6 \mathrm{~Hz}, \mathrm{H}-18), 2.16$, 2.09, 2.05, $2.00\left(\mathrm{~s}, 4 \times 3 \mathrm{H}, \underline{\mathrm{CH}}_{3} \mathrm{CO}\right), 1.11,1.00,0.92,0.89,0.89,0.82,0.60\left(\mathrm{~s}, 7 \times 3 \mathrm{H}, \mathrm{CH}_{3}\right) ;{ }^{13} \mathrm{C}-$ NMR $\left(\mathrm{CDCl}_{3}\right): \delta 177.4,170.6,170.2,169.9,169.8(5 \mathrm{C}=\mathrm{O}), 143.6,136.3,128.3,128.3,127.9,127.9$, 122.4, 94.6 (C-1'), 84.7, 77.2, 70.7, 69.2, 69.0, 66.4, 66.3, 65.9, 62.6, 55.6, 47.6, 46.7, 45.8, 41.6, 41.3, $39.3,38.3,38.0,36.8,33.8,33.0,32.7,32.3,30.6,28.7,27.6,25.8,23.6,23.4,23.0,22.1,20.8,20.6$, 20.6, 18.2, 16.8, 16.4, 15.2; HRESIMS: $m / z$ calcd. for $\mathrm{C}_{51} \mathrm{H}_{72} \mathrm{O}_{8} \mathrm{Na}\left[\mathrm{M}+\mathrm{Na}^{+}\right]$: 835.5125; found: $\mathrm{m} / z$ 835.5118 .

Oleanolic acid 3-O- $\alpha$-D-mannopyranoside (4). Compound 4 was prepared from $\mathbf{3 6}$ by the same procedure as for 1. Yield: 87\%; white powder, m.p. $250-252{ }^{\circ} \mathrm{C}, R_{\mathrm{f}}=0.07\left(20: 1: 0.1 \mathrm{CHCl}_{3}-\mathrm{MeOH}-\right.$ $\left.\mathrm{H}_{2} \mathrm{O}\right) ;[\alpha]_{\mathrm{D}}^{25}+79.8(c 0.5, \mathrm{MeOH}) ;{ }^{1} \mathrm{H}-\mathrm{NMR}\left(\right.$ pyridine- $\left._{5}\right): \delta 5.54\left(\mathrm{~d}, 1 \mathrm{H}, J=1.0 \mathrm{~Hz}, \mathrm{H}-1^{\prime}\right), 5.46$ (br s, $1 \mathrm{H}, \mathrm{H}-12), 4.69$ (m, $1 \mathrm{H}), 4.59-4.50$ (m, $3 \mathrm{H}), 4.46-4.38$ (m, $2 \mathrm{H}), 3.47$ (dd, $1 \mathrm{H}, J=4.2,11.4 \mathrm{~Hz}, \mathrm{H}-$ 3), 3.28 (dd, $1 \mathrm{H}, J=4.0,13.5 \mathrm{~Hz}, \mathrm{H}-18), 1.24,1.15,1.00,0.97,0.94,0.81,0.79$ (s, $7 \times 3 \mathrm{H}, \mathrm{CH}_{3}$ ); ${ }^{13} \mathrm{C}-\mathrm{NMR}$ (pyridine- $\mathrm{d}_{5}$ ): $\delta 180.1(\mathrm{C}=\mathrm{O}), 144.8,124.1,122.4,97.7(\mathrm{C}-1$ ') $, 81.8,75.8,73.2,72.9,69.2$, 63.4, 55.7, 47.9, 46.6, 46.4, 42.1, 41.9, 39.7, 38.5, 38.1, 37.1, 34.2, 33.2, 33.1, 33.1, 30.9, 29.0, 28.2, 
26.1, 23.7, 23.6, 22.0, 18.5, 17.3, 16.9, 15.3; HRESIMS: $m / z$ calcd. for $\mathrm{C}_{36} \mathrm{H}_{58} \mathrm{O}_{8} \mathrm{Na}\left[\mathrm{M}+\mathrm{Na}^{+}\right]$: 641.4029; found: $m / z 641.4037$.

Benzyl oleanolate 3-O-2,3,4-tri-O-benzoyl- $\beta$-D-xylopyranosyl- $(1 \rightarrow 4)-2,3$-di-O-benzoyl-6-deoxy- $\alpha$ - $L$ talopyranoside (38). Compound 16 (1.1 g, $1.3 \mathrm{mmol})$ and 14 [13] (0.9 g, $1.5 \mathrm{mmol})$ were coupled under the same conditions as that used for the preparation of $\mathbf{2 3}$ from $\mathbf{1 1}$ and $\mathbf{9}$, giving $\mathbf{3 8}$ (1.4 g, 90\%) as a foamy solid. $R_{\mathrm{f}}=0.13\left(8: 1\right.$ petroleum ether-EtOAc); $[\alpha]_{\mathrm{D}}^{25}-24.6\left(c 0.5, \mathrm{CHCl}_{3}\right) ;{ }^{1} \mathrm{H}-\mathrm{NMR}\left(\mathrm{CDCl}_{3}\right)$ : $\delta$ 8.33-7.26 (m, $30 \mathrm{H}, \mathrm{Ar}-\underline{\mathrm{H}}$ ), 5.67 (dd, $\left.1 \mathrm{H}, J=8.2,9.1 \mathrm{~Hz}, \mathrm{H}-2^{\prime \prime}\right), 5.58$ (dd, $\left.1 \mathrm{H}, J=6.7,9.1 \mathrm{~Hz}, \mathrm{H}-3^{\prime \prime}\right)$, 5.48 (d, $\left.1 \mathrm{H}, J=2.2 \mathrm{~Hz}, \mathrm{H}-1^{\prime}\right), 5.40$ (dd, 1 H, J=3.6, 3.6 Hz, H-3'), 5.28 (br s, $1 \mathrm{H}, \mathrm{H}-12$ ), 5.07 (dd, 2 $\left.\mathrm{H}, J=12.5,17.6 \mathrm{~Hz}, \mathrm{PhCH}_{2}\right), 4.98-4.92$ (m, $\left.2 \mathrm{H}\right), 4.70$ (d, $\left.1 \mathrm{H}, J=6.7 \mathrm{~Hz}, \mathrm{H}-1 "\right), 4.29-4.19$ (m, $2 \mathrm{H}$ ), 3.50 (m, $1 \mathrm{H}), 3.14-3.04$ (m, 2 H), 2.89 (dd, 1 H, $J=4.1,9.6$ Hz, H-18), 1.19 (d, 3 H, $J=6.5$ Hz, H-6'), 1.10, 0.97, 0.91, 0.89, 0.89, 0.81, $0.59\left(\mathrm{~s}, 7 \times 3 \mathrm{H}, \mathrm{CH}_{3}\right) ;{ }^{13} \mathrm{C}-\mathrm{NMR}\left(\mathrm{CDCl}_{3}\right): \delta 177.4,166.3,166.2$, 165.6, 165.2, $164.9(6 \mathrm{C}=\mathrm{O}), 143.7,140.9,138.8,136.4,133.3,133.2,133.1,133.0,130.4,130.0$, 130.0, 129.9, 129.8, 129.7, 129.2, 129.1, 128.6, 128.5, 128.4, 128.4, 128.3, 128.3, 128.3, 128.0, 127.9, 127.8, 127.6, 127.5, 126.9, 122.4, 103.1, 100.7 (2× C-1), 99.4, 89.5, 78.1, 77.3, 76.6, 76.4, 74.2, 73.7, $72.8,72.3,71.8,71.6,69.9,68.6,68.3,65.9,65.6,65.3,62.1,60.2,55.5,55.4,47.5,46.7,45.9,41.7$, 41.4, 39.3, 38.9, 38.4, 36.7, 33.1, 32.4, 30.7, 28.3, 27.6, 25.8, 23.6, 23.4, 23.1, 18.2, 16.9, 16.5, 16.1, 15.3; HRESIMS: $m / z$ calcd. for $\mathrm{C}_{83} \mathrm{H}_{92} \mathrm{O}_{16} \mathrm{Na}\left[\mathrm{M}+\mathrm{Na}^{+}\right]$: 1367.6283 ; found: $m / z$ 1367.6290.

Oleanolic acid 3-O- $\beta$-D-xylopyranosyl-( $1 \rightarrow 4)$-6-deoxy- $\alpha$-L-talopyranoside (5). Compound 5 was prepared from 38 by the same procedure as for 1 . Yield: $71 \%$; white powder, m.p. 218-220 ${ }^{\circ} \mathrm{C}$, $R_{\mathrm{f}}=0.11\left(10: 1: 0.1 \mathrm{CHCl}_{3}-\mathrm{MeOH}-\mathrm{H}_{2} \mathrm{O}\right) ;[\alpha]_{\mathrm{D}}^{25}-30.7\left(c\right.$ 0.5, MeOH); ${ }^{1} \mathrm{H}-\mathrm{NMR}\left(\right.$ pyridine- $\left.\mathrm{d}_{5}\right): \delta 5.47(\mathrm{br}$ s, $1 \mathrm{H}, \mathrm{H}-12), 5.27$ (s, $\left.1 \mathrm{H}, \mathrm{H}-1^{\prime}\right), 4.80$ (d, $\left.1 \mathrm{H}, J=7.4 \mathrm{~Hz}, \mathrm{H}-1 "\right), 4.34-4.30$ (m, $2 \mathrm{H}$ ), 4.25-4.20 (m, $3 \mathrm{H}), 4.13-3.93$ (m, $3 \mathrm{H}), 3.69$ (dd, $1 \mathrm{H}, J=9.6,10.9 \mathrm{~Hz}), 3.29$ (dd, $1 \mathrm{H}, J=3.9,13.6 \mathrm{~Hz}, \mathrm{H}-3), 3.11$ (dd, $1 \mathrm{H}, J=4.3,11.6 \mathrm{~Hz}, \mathrm{H}-18$ ), 1.70 (d, $3 \mathrm{H}, J=6.6 \mathrm{~Hz}, \mathrm{H}-6$ '), 1.29, 1.00, 1.00, 0.95, 0.92, 0.84, 0.79 $\left(\mathrm{s}, 7 \times 3 \mathrm{H}, \mathrm{CH}_{3}\right) ;{ }^{13} \mathrm{C}-\mathrm{NMR}\left(\right.$ pyridine-d $\left._{5}\right): \delta 180.1(\mathrm{C}=\mathrm{O}), 144.8,122.4,106.3,104.9(2 \times \mathrm{C}-1), 88.6$, 83.3, 77.8, 74.7, 71.9, 70.5, 67.2, 67.1, 66.7, 55.5, 47.9, 46.6, 46.4, 42.1, 42.0, 39.7, 39.1, 38.4, 36.9, 34.2 , 33.2, 33.2, 33.1, 30.9, 28.2, 26.1, 25.6, 23.7, 23.7, 23.7, 23.6, 18.5, 17.3, 17.0, 16.6, 15.4; HRESIMS: $m / z$ calcd. for $\mathrm{C}_{41} \mathrm{H}_{66} \mathrm{O}_{11} \mathrm{Na}\left[\mathrm{M}+\mathrm{Na}^{+}\right]$: 757.4503 ; found: $m / z$ 757.4515.

Benzyl oleanolate 3-O-2,3,4,6-tetra-O-benzoyl- $\beta$-D-galactopyranosyl-(1 $\rightarrow 4)$-2,3-di-O-benzoyl- $\alpha$ - $L$ rhamnopyranoside (40). Compound 12 [11] (0.9 g, $1.2 \mathrm{mmol})$ and 31 (0.9 g, $1.0 \mathrm{mmol})$ were coupled under the same conditions as that used for the preparation of 23 from $\mathbf{1 1}$ and $\mathbf{9}$, giving $\mathbf{4 0}$ (1.3 g, 89\%) as a foamy solid. $R_{\mathrm{f}}=0.07$ (8:1 petroleum ether-EtOAc); $[\alpha]_{\mathrm{D}}^{25}+64.5\left(c \quad 0.5, \mathrm{CHCl}_{3}\right) ;{ }^{1} \mathrm{H}-\mathrm{NMR}$ $\left(\mathrm{CDCl}_{3}\right): \delta$ 8.07-7.05 (m, $\left.35 \mathrm{H}, \mathrm{Ar}-\underline{\mathrm{H}}\right), 5.97$ (d, $\left.1 \mathrm{H}, J=3.1 \mathrm{~Hz}, \mathrm{H}-4 "\right), 5.75$ (dd, $1 \mathrm{H}, J=7.9,10.4 \mathrm{~Hz}$, H-2"), 5.55-5.47 (m, 2 H, H-2', H-3'), 5.41 (dd, 1 H, J=3.3, 10.4 Hz, H-3"), 5.30 (br s, 1 H, H-12), 5.13-5.07 (m, 3 H, H-1", $\mathrm{PhCH}_{2}$ ), 4.94 (d, $1 \mathrm{H}, J=1.4 \mathrm{~Hz}, \mathrm{H}-1$ '), 4.71-4.37 (m, $3 \mathrm{H}$ ), 4.21-4.05 (m, $2 \mathrm{H}), 3.14(\mathrm{dd}, 1 \mathrm{H}, J=7.5,8.6 \mathrm{~Hz}, \mathrm{H}-3), 2.91(\mathrm{dd}, 1 \mathrm{H}, J=3.9,13.7 \mathrm{~Hz}, \mathrm{H}-18), 1.50(\mathrm{~d}, 1 \mathrm{H}$, $J=6.0 \mathrm{~Hz}, \mathrm{H}-6$ '), 1.12, 0.96, 0.92, 0.92, 0.89, 0.85, $0.61\left(\mathrm{~s}, 7 \times 3 \mathrm{H}, \mathrm{CH}_{3}\right) ;{ }^{13} \mathrm{C}-\mathrm{NMR}\left(\mathrm{CDCl}_{3}\right): \delta 177.4$, 166.1, 165.5, 165.4, 165.4, 165.3, 164.7 (7 C=O), 143.7, 136.4, 133.5, 133.3, 133.2, 133.1, 132.8, $129.9,129.7,129.7,129.7,129.6,129.6,129.6,129.6,129.6,129.5,129.5,129.4,129.3,129.0,128.8$, 
$128.6,128.6,128.5,128.5,128.4,128.4,128.4,128.4,128.4,128.4,128.4,128.4,128.3,128.2,128.1$, $128.0,128.0,128.0,128.0,127.9,122.5,101.5,99.8(2 \times \mathrm{C}-1), 89.9,77.6,77.2,72.5,71.9,71.1,70.9$, 69.7, 68.0, 67.1, 65.9, 62.0, 55.4, 47.6, 46.7, 45.9, 41.7, 41.4, 39.3, 38.9, 38.5, 36.7, 33.8, 33.0, 32.7, 32.4, 30.6, 28.3, 27.6, 25.8, 25.4, 23.6, 23.4, 23.1, 18.2, 18.1, 16.8, 16.5, 15.3; HRESIMS: $\mathrm{m} / z$ calcd. for $\mathrm{C}_{91} \mathrm{H}_{98} \mathrm{O}_{18} \mathrm{Na}\left[\mathrm{M}+\mathrm{Na}^{+}\right]$: 1501.6651 ; found: $m / z$ 1501.6629.

Oleanolic acid 3-O- $\beta$-D-galactopyranosyl-(1 $\rightarrow 4)$ - $\alpha$-L-rhamnopyranoside (6). Compound 6 was prepared from 40 by the same procedure as for 1 . Yield: $68 \%$; white powder, m.p. $268-270{ }^{\circ} \mathrm{C}$, $R_{\mathrm{f}}=0.70\left(10: 2: 0.1 \mathrm{CHCl}_{3}-\mathrm{MeOH}-\mathrm{H}_{2} \mathrm{O}\right) ;[\alpha]_{\mathrm{D}}^{25}+24.6\left(c\right.$ 0.5, MeOH); ${ }^{1} \mathrm{H}-\mathrm{NMR}\left(\right.$ pyridine- $\left.\mathrm{d}_{5}\right): \delta 5.46(\mathrm{br}$ s, 1 H, H-12), 5.27 (s, 1 H, H-1'), 5.18 (d, 1 H, J= 7.8 Hz, H-1"), 4.60-4.24 (m, 8 H), 4.14 (dd, 1 H, $J=3.4,9.5 \mathrm{~Hz}$ ), 3.95 (dd, $1 \mathrm{H}, J=6.1,6.4 \mathrm{~Hz}, \mathrm{H}-2 "), 3.29$ (dd, $1 \mathrm{H}, J=4.2,13.7 \mathrm{~Hz}, \mathrm{H}-3$ ), 3.11 (dd, 1 $\mathrm{H}, J=4.5,11.6 \mathrm{~Hz}, \mathrm{H}-18$ ), 1.74 (d, $3 \mathrm{H}, J=6.2 \mathrm{~Hz}, \mathrm{H}-6$ '), 1.27, 1.00, 0.99, 0.95, 0.89, 0.83, 0.76 (s, $\left.7 \times 3 \mathrm{H}, \mathrm{CH}_{3}\right) ;{ }^{13} \mathrm{C}-\mathrm{NMR}\left(\right.$ pyridine- $\left.\mathrm{d}_{5}\right): \delta 180.1(\mathrm{C}=\mathrm{O}), 144.8,122.7,122.5,107.3,103.9(2 \times \mathrm{C}-1)$, 88.5, 85.2, 76.9, 75.6, 74.1, 72.9, 71.9, 70.0, 68.1, 62.0, 55.5, 47.9, 46.6, 46.4, 42.1, 42.0, 39.7, 39.1, 38.4, 36.9, 34.2, 33.2, 33.2, 33.1, 30.9, 28.3, 28.2, 26.1, 25.7, 23.7, 23.7, 18.5, 18.3, 17.3, 16.7, 15.4; HRESIMS: $m / z$ calcd. for $\mathrm{C}_{42} \mathrm{H}_{68} \mathrm{O}_{12} \mathrm{Na}\left[\mathrm{M}+\mathrm{Na}^{+}\right]$: 787.4608 ; found: $m / z$ 787.4579.

Benzyl oleanolate 3-O-2,3,4,6-tetra-O-benzoyl- $\beta$-D-galactopyranosyl-(1 $\rightarrow 3)$-2,4-di-O-benzoyl- $\beta$ - $D$ -

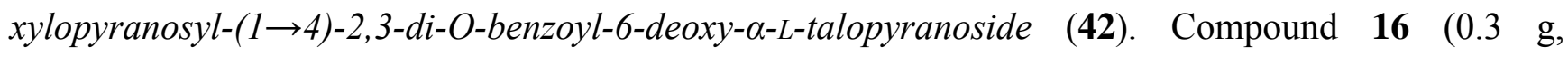
$0.3 \mathrm{mmol})$ and $15(0.41 \mathrm{~g}, 0.4 \mathrm{mmol})$ were coupled under the same conditions as used for the preparation of 23 from 11 and 9, giving $42(0.5 \mathrm{~g}, 90 \%)$ as a foamy solid. $R_{\mathrm{f}}=0.11$ (4:1 petroleum ether-EtOAc); $[\alpha]_{\mathrm{D}}^{25}-26.6\left(c \quad 0.5, \mathrm{CHCl}_{3}\right) ;{ }^{1} \mathrm{H}-\mathrm{NMR}\left(\mathrm{CDCl}_{3}\right): \delta 8.28-7.16(\mathrm{~m}, 45 \mathrm{H}, \mathrm{Ar}-\underline{\mathrm{H}}), 5.78(\mathrm{~d}$, $1 \mathrm{H}, J=3.5 \mathrm{~Hz}), 5.59(\mathrm{dd}, 1 \mathrm{H}, J=7.9,10.4 \mathrm{~Hz}), 5.47-5.41(\mathrm{~m}, 2 \mathrm{H}), 5.26-5.20(\mathrm{~m}, 2 \mathrm{H}), 5.06(\mathrm{dd}$, $\left.2 \mathrm{H}, J=12.5,17.3 \mathrm{~Hz}, \mathrm{PhC}_{2}\right), 4.93-4.86(\mathrm{~m}, 3 \mathrm{H}), 4.44$ (d, $\left.1 \mathrm{H}, J=1.4 \mathrm{~Hz}\right), 4.39-3.92$ (m, $\left.6 \mathrm{H}\right), 3.30$ (dd, $1 \mathrm{H}, J=5.6,12.1 \mathrm{~Hz}, \mathrm{H}-3), 3.06-2.87$ (m, $3 \mathrm{H}), 0.99$ (d, $1 \mathrm{H}, J=6.5 \mathrm{~Hz}, \mathrm{H}-6$ '), 1.09, 0.93, 0.91, $0.89,0.86,0.77,0.58\left(\mathrm{~s}, 7 \times 3 \mathrm{H}, \mathrm{CH}_{3}\right) ;{ }^{13} \mathrm{C}-\mathrm{NMR}\left(\mathrm{CDCl}_{3}\right) \delta 177.4,166.3,166.2,165.8,165.4,165.3$, 165.0, 164.9, 163.9 (9 C=O), 143.7, 136.4, 133.3, 133.3, 133.3, 133.2, 133.2, 133.1, 133.0, 132.9, $130.5,130.0,129.9,129.9,129.8,129.8,129.7,129.7,129.7,129.7,129.7,129.7,129.6,129.6,129.6$, $129.5,129.5,129.4,129.4,129.4,129.4,129.2,129.0,128.7,128.6,128.5,128.5,128.5,128.5,128.5$, $128.4,128.4,128.4,128.4,128.4,128.4,128.3,128.3,128.2,128.2,128.1,128.0,127.9,127.9,127.8$, $127.8,122.4,103.3,101.0,100.6(3 \times \mathrm{C}-1), 89.3,77.3,73.2,71.7,70.9,70.2,69.7,68.5,68.0,67.5$, $65.9,65.4,62.4,61.2,55.3,47.5,46.7,45.9,41.7,41.4,39.3,38.9,38.3,36.6,33.8,33.0,32.6,32.3$, 30.6, 28.2, 27.6, 25.8, 25.1, 23.6, 23.3, 23.0, 18.2, 16.8, 16.4, 15.9, 15.3; HRESIMS: $m / z$ calcd. for $\mathrm{C}_{110} \mathrm{H}_{114} \mathrm{O}_{24} \mathrm{Na}\left[\mathrm{M}+\mathrm{Na}^{+}\right.$]: 1841.7598 ; found: $m / z$ 1841.7579.

Oleanolic acid 3-O- $\beta$-D-galactopyranosyl-( $1 \rightarrow 3)$ - $\beta$-D-xylopyranosyl- $(1 \rightarrow 4)-6$-deoxy- $\alpha$-L-talopyranoside (7). Compound 7 was prepared from 42 by the same procedure as for $\mathbf{1}$. Yield: 75\%; white powder, m.p. $202-204{ }^{\circ} \mathrm{C}, R_{\mathrm{f}}=0.82\left(10: 2: 0.1 \mathrm{CHCl}_{3}-\mathrm{MeOH}-\mathrm{H}_{2} \mathrm{O}\right) ;[\alpha]_{\mathrm{D}}^{25}-36.8(c 0.5, \mathrm{MeOH}) ;{ }^{1} \mathrm{H}-$ NMR (pyridine-d $)_{5}$ ): $\delta 5.47$ (br s, $\left.1 \mathrm{H}, \mathrm{H}-12\right), 5.27-5.25$ (m, $2 \mathrm{H}$ ), 4.72 (d, $1 \mathrm{H}, J=7.8 \mathrm{~Hz}$ ), 4.54-4.50 (m, $2 \mathrm{H}), 4.40-3.91(\mathrm{~m}, 12 \mathrm{H}), 3.85(\mathrm{dd}, 1 \mathrm{H}, J=7.0,7.0 \mathrm{~Hz}), 3.59$ (dd, $1 \mathrm{H}, J=10.2,11.2 \mathrm{~Hz}), 3.28$ (dd, $1 \mathrm{H}, J=4.2,10.0 \mathrm{~Hz}, \mathrm{H}-3$ ), 3.09 (dd, $1 \mathrm{H}, J=4.4,11.4 \mathrm{~Hz}, \mathrm{H}-18$ ), 1.67 (d, $3 \mathrm{H}, J=6.5 \mathrm{~Hz}, \mathrm{H}-6$ '), 
1.28, 1.00, 0.99, 0.95, 0.90, 0.83, $0.78\left(\mathrm{~s}, 7 \times 3 \mathrm{H}, \mathrm{CH}_{3}\right) ;{ }^{13} \mathrm{C}-\mathrm{NMR}$ (pyridine- $\left.\mathrm{d}_{5}\right): \delta 180.2,144.9$, 122.5, 106.3, 105.9, $104.9(3 \times \mathrm{C}-1), 88.8,86.8,83.6,77.3,75.2,73.5,73.1,72.0,70.2,69.0,67.1$, 66.7, 66.5, 62.1, 57.4, 55.6, 48.0, 46.7, 46.5, 42.2, 42.1, 39.8, 39.2, 38.5, 37.0, 34.3, 33.3, 33.2, 31.0, 28.4, 28.3, 26.2, 25.7, 23.8, 23.7, 19.2, 18.6, 17.4, 17.0, 16.8, 15.5; HRESIMS: $\mathrm{m} / \mathrm{z}$ calcd. for $\mathrm{C}_{47} \mathrm{H}_{76} \mathrm{O}_{16} \mathrm{Na}\left[\mathrm{M}+\mathrm{Na}^{+}\right]$: 919.5031 ; found: $m / z$ 919.5018.

\subsection{Fungicidal activity bioassay}

We used the mycelium growth rate test [20]. The culture media, with known concentration of the test compounds, were obtained by mixing the soln of compounds 1-7 in methanol with potato dextrose agar (PDA), on which fungus cakes were placed. The blank test was made using methanol. The culture was carried out at $24 \pm 0.5^{\circ} \mathrm{C}$. Three replicates were performed.

\section{Conclusions}

Seven glycoconjugates of oleanolic acid were designed and efficiently synthesized. The bioassays showed that they had some fungicidal activity against four fungi. All of the compounds exhibited more fungicidal activity against $R$. solani, and the compounds $\mathbf{1}$ and $\mathbf{2}$ had better activity against $B$. cinerea and $P$. CapasiciLeonian than the other compounds.

\section{Acknowledgements}

This work was supported by the NSFC (No. 20902108) of China, National Basic Research Program of China (No. 2010CB126105), and the National Scientific \& Technical Supporting Programs (No. 2009BAK61B04).

\section{References and Notes}

1. Papadopoulou, K.; Melton, R.E.; Legget, M.; Daniels, M.J.; Osbourn, A.E. Compromised disease resistance in saponin-deficient plants. Proc. Natl. Acad. Sci. USA 1999, 96, 12923-12928.

2. Mahato, S.B.; Garai, S. Triterpenoid saponins. Progr. Chem. Org. Nat. Prod. 1998, 74, 1-196.

3. Gauthier, C.; Legault, J.; Pichette, A. Recent progress in the synthesis of naturally occurring triterpenoid saponins. Mini-Rev. Org. Chem. 2009, 6, 321-344.

4. Yu, B.; Sun, J. Current synthesis of triterpene saponins. Chem. Asian J. 2009, 4, 642-654.

5. Paczkowski, C.; Wojciechowski, Z.A. Glucosylation and galactosylation of diosgenin and solasodine by soluble glycosyltransferase(s) from Solanum melongena leaves. Phytochemistry 1994, 35, 1429-1434.

6. Wojciechowski, Z.A. Biosynthesis of oleanolic acid glycosides by subcellular fractions of Calendula officinalis seedlings. Phytochemistry 1975, 14, 1749-1753.

7. Hostettmann, K.; Marton, A. Saponins. Chemistry and Pharmacology of Natural Products; Cambridge University Press: Cambridge, UK, 1995; p. 560.

8. Yadava, R.N.; Jharbade, J. A new bioactive triterpenoid saponin from the seeds of Lactuca scariola Linn. Nat. Prod. Res. Part A: Struct. Synth. 2007, 21, 500-506. 
9. Schmidt, R.R.; Kinzy, W. Anomeric-oxygen activation for glycoside synthesis: the trichloroacetimidate method. Adv. Carbohydr. Chem. Biochem. 1994, 50, 21-123.

10. Chen, L.; Zhang, Y.H.; Kong, X.G.; Lan, E.; Huang, Z.J.; Peng, S.X.; Kaufman, D.L.; Tian, J.D. Design, synthesis, and antihepatocellular carcinoma activity of nitric oxide releasing derivatives of oleanolic acid. J. Med. Chem. 2008, 51, 4834-4838.

11. Lafont, D.; Bouchu, M.N.; Girard-Egrot, A.; Boullanger, P. Syntheses and interfacial behavior of neoglycolipid analogues of glycosyl ceramides. Carbohydr. Res. 2001, 336, 181-194.

12. Kimmel, R.; Kafka, S.; Kosmrlj, J. Selective formation of glycosidic linkages of N-unsubstituted 4-hydroxyquinolin-2-(1H)-ones. Carbohydr. Res. 2010, 345, 768-779.

13. Sha, Y.; Yan, M.C.; Liu, J.; Liu, Y.; Cheng, M.S. Facile synthesis of oleanolic acid monoglycosides and diglycosides. Molecules 2008, 13, 1472-1486.

14. Sarkar, K.; Mukherjee, I.; Roy, N. Synthesis of the trisaccharide repeating unit of the O-antigen related to the enterohemorrhagic Escherichia coli type $\mathrm{O}_{26}: H$. J. Carbohydr. Chem. 2003, 22, 95-107.

15. Yan, S.Q.; Wu, X.M.; Liang, X.M.; Zhang, J.J.; Wang, D.Q. Synthesis of di- and tetrasaccharide containing 6-deoxy-L-talose from the O-antigenic polysaccharide of Burkholderia pseudomallei strain 304b. Chin. Chem. Lett. 2009, 20, 582-585.

16. Agarwal, A.; Vankar, Y.D. Selective deprotection of terminal isopropylidene acetals and trityl ethers using $\mathrm{HClO}_{4}$ supported on silica gel. Carbohydr. Res. 2005, 340, 1661-1667.

17. Zhong, Y.L.; Shing, T.K.M. Efficient and Facile Glycol Cleavage Oxidation Using Improved Silica Gel-Supported Sodium Metaperiodate. J. Org. Chem. 1997, 62, 2622-2624.

18. Zhang, J.J.; Liang, X.M.; Wang, D.Q.; Kong, F.Z. Regio- and stereoselective anomeric esterification of glucopyranose 1,2-diols and a facile preparation of 2-O-acetylated glucopyranosyl trichloroacetimidates from the corresponding 1,2-diols. Carbohydr. Res. 2007, 342, 797-805.

19. Guo, T.; Liu, Q.; Wang, P.; Zhang, L.; Zhang, W.; Li, Y. Facile synthesis of three bidesmosidic oleanolic acid saponins with strong inhibitory activity on pancreatic lipase. Carbohydr. Res. 2009, 344, 1167-1174.

20. Chen, N.C. Bioassay of Pesticides; Beijing Agricultural University Press: Beijing, China, 1991; pp. 161-162.

Sample Availability: Samples of the compounds are available from the authors.

(C) 2011 by the authors; licensee MDPI, Basel, Switzerland. This article is an open access article distributed under the terms and conditions of the Creative Commons Attribution license (http://creativecommons.org/licenses/by/3.0/). 